\title{
Improving the boundary efficiency of a compact finite difference scheme through optimising its composite template
}

\author{
Jacob M. Turner*, Sina Haeri**, Jae Wook Kim \\ Aerodynamics and Flight Mechanics Research Group, University of Southampton, \\ Southampton, SO17 1BJ, UK
}

\begin{abstract}
This paper presents efforts to improve the boundary efficiency and accuracy of a compact finite difference scheme, based on its composite template. Unlike precursory attempts the current methodology is unique in its quantification of dispersion and dissipation errors, which are only evaluated after the matrix system of equations has been rearranged for the derivative. This results in a more accurate prediction of the boundary performance, since the analysis is directly based on how the derivative is represented in simulations. A genetic algorithm acts as a comprehensive method for the optimisation of the boundary coefficients, incorporating an eigenvalue constraint for the linear stability of the matrix system of equations. The performance of the optimised composite template is tested on one-dimensional linear wave convection and two-dimensional inviscid vortex convection problems, with uniform and curvilinear grids. In all cases, it yields substantial accuracy and

\footnotetext{
*Corresponding author, Email address: J.M.Turner@soton.ac.uk

** Current address: Department of Mechanical and Aerospace Engineering, University of Strathclyde, Glassgow, G1 1XQ, UK
} 
efficiency improvements while maintaining stable solutions and fourth-order accuracy.

Keywords: Compact finite difference; Boundary closure; Optimization; Genetic algorithm; Composite template

\section{Introduction}

Compact finite differences are numerical schemes used to accurately calculate derivatives. They are implicit in nature, based upon a banded Hermitian matrix system of equations. Although inverting such a system requires a higher computational cost, they can offer vastly superior resolution for a given stencil size compared to their explicit counterparts. This quality has made them increasingly popular in the fields of computational aeroacoustics (CAA) $[1,2]$, large eddy simulation (LES) [3-5], and direct numerical simulation (DNS) [6-8], particularly when high resolution is a necessity in order to properly resolve the relevant physical scales.

Typically, central differences are used to construct compact schemes for use at interior nodes. However, such schemes are not always applicable at domain boundaries, and therefore in order to properly close the matrix system of equations non-central differences are often a necessity. This unfortunately will have a detrimental effect on accuracy; introducing additional dissipation as well as dispersion, if the boundary schemes are not sufficiently optimised. Consequently, to ensure that the same level of accuracy is achieved throughout the entire domain, grid refinements are regularly made to the boundary regions. This will inevitably reduce computational efficiency due to the decreased time step required by the smaller grid cells. The objective 
of this paper is to build upon past attempts to maximise boundary scheme performance, and thereby minimise efficiency losses, while also ensuring the combination of interior and boundary schemes meets requirements for linear stability.

As well as changes in formal order of accuracy, enhancements to compact schemes can also be achieved through coefficient optimisation based on resolution characteristics. A previous attempt at this was undertaken by Kim [1]. Kim introduced a highly optimised fourth-order pentadiagonal compact scheme and set of boundary closures particularly for CAA applications. Optimisations were based on an integral error measure between the exact and modified wavenumber solutions (similar to Kim and Lee [9]). Very low resolution errors were obtained with this method, in particular for the interior scheme, which remains below $0.1 \%$ over the grid spaced scaled wavenumber range $0 \leq \omega \leq 0.839 \pi$. The boundary schemes were designed to maintain the same stencil size and order of accuracy as the interior schemes, which was accomplished by employing extrapolation functions based on both polynomial and trigonometric series for solutions outside of the domain. After some algebraic manipulation, these were then converted into a set of noncentral differences for use at the domain boundaries. The resultant boundary schemes were optimised by means of control variables left open in the trigonometric series of each extrapolation function. As in Carpenter et al. [10] the linear stability of the matrix system was investigated using eigenvalue analysis. Kim [1] found that with a coarse grid the schemes contained some slightly positive eigenvalue components. Although, after some grid refinement it was demonstrated that these will tend towards zero, hence implying 
neutral stability.

Liu et al. [11] expanded on the optimisation strategy of Kim [1] by introducing a sequential quadratic programming technique (SQP). This iteratively increased the upper limit of the optimisation range $(r)$, establishing optimal values for both interior and boundary schemes. Furthermore, they showed that scheme stability is heavily dependent on the chosen error tolerances, as well as the formal order of accuracy, implying that the optimisation process can often be detrimental to the numerical stability. To compensate for this, Liu et al. [11] reduced the order of accuracy of their first and third boundary schemes by one stage. Such stability issues were also recognised by Carpenter et al. [10], who suggested that a scheme's numerical stability and its spectral resolution do not always coincide.

Jordan [12] introduced an alternative approach for analysing spectral resolution properties through composite templates. Unlike the more traditional decoupled Fourier approach where the resolution of each differencing stencil is studied separately, this consists of Fourier analysis of the whole matrix system of equations, consisting of both the interior and boundary stencils. The result is a set of pseudo-wavenumber curves for each point in the grid, dependent on the number of grid points used in the analysis. Jordan applied this analysis to tridiagonal systems, employing a least squares optimisation strategy to minimise the total resolution error across the whole template. In a later paper by Jordan [13] the same technique was applied to pentadiagonal systems producing a set a of boundary closure schemes to be used alongside the interior scheme of Kim [1]. Although the modified wavenumber curves produced by this technique are dependent on the number of grid points used 
in the analysis, they appear to be much more representative of the performance we achieve once schemes are applied to actual simulations. Despite this, it is still unclear how to best optimise the resolution properties of a given composite template, making it far from a trivial task. For instance one could prioritise minimising the relative resolution error between neighbouring points in the composite template, or perhaps the aggregate resolution error of the whole template with respect to the exact wavenumber.

This paper aims to extend the composite template strategy of Jordan [12] by redefining how the composite template modified wavenumber is evaluated. Unlike the original approach, Fourier analysis will not be conducted until the matrix system of equations has already been rearranged for the derivative. This should lead to better predictions of the resolution properties attained in simulations because this is a closer depiction of how the derivative is represented numerically. The chosen optimisation method is a Genetic Algorithm (GA) containing both an objective function for the composite template's resolution characteristics, and a non-linear constraint for eigenvalue stability. In this paper, the optimisation procedure is applied to the pentadiagonal finite-difference system outlined by Kim [1], although a similar approach would be applicable to other systems if desired. The newly optimised boundary closure coefficients are successful in producing large accuracy improvements while maintaining stable solutions in all test problems. In addition to the primary optimisation which focuses on the aggregate resolution error of the composite template, further accuracy enhancements are attempted by introducing pseudo-boundary schemes. Essentially these are tuned central schemes applied as intermediate steps between the boundary 
and interior regions, with the aim of reducing the relative resolution error between consecutive points. They are successful in achieving further accuracy improvements, albeit with some penalty to numerical stability.

The paper is organised as follows. Section 2 introduces the compact finite-difference system, and outlines the new composite template modified wavenumber analysis. Section 3 provides details of the boundary closure scheme coefficient optimisation procedure. Including the optimisation platform, objective function and stability constraints. Section 4 presents the optimisation results, including the resultant wavenumber characteristics and eigenvalue distribution. In section 5 the performance of the newly optimised finite-difference system is tested in three benchmark problems, designed to analyse their performance in a variety of scenarios. In section 6 pseudoboundary schemes are introduced and their performance analysed. Finally concluding remarks are given in section 7 .

\section{Compact Finite Difference Schemes and Composite Template Modified Wavenumber Analysis}

We consider the following general compact finite difference template, based on a pentadiagonal Hermitian matrix. It is constructed from one central interior and three non-central boundary closure schemes, each in conservative form and utilising a seven-point stencil [1].

$$
\mathbf{P} \overline{\mathbf{f}}^{\prime}=\frac{1}{h} \mathbf{Q f}
$$

where $\mathbf{P}$ and $\mathbf{Q}$ are the following $(N+1) \times(N+1)$ matrices 


$$
\begin{aligned}
& \boldsymbol{P}=\left(\begin{array}{ccccccccc}
1 & \gamma_{01} & \gamma_{02} & 0 & \cdots & 0 & 0 & 0 & 0 \\
\gamma_{10} & 1 & \gamma_{12} & \gamma_{13} & 0 & \cdots & 0 & 0 & 0 \\
\gamma_{20} & \gamma_{21} & 1 & \gamma_{23} & \gamma_{24} & 0 & \cdots & 0 & 0 \\
0 & \beta & \alpha & 1 & \alpha & \beta & 0 & \cdots & 0 \\
\vdots & \ddots & \ddots & \ddots & \ddots & \ddots & \ddots & \ddots & \vdots \\
0 & \cdots & 0 & \beta & \alpha & 1 & \alpha & \beta & 0 \\
0 & 0 & \cdots & 0 & \gamma_{24} & \gamma_{23} & 1 & \gamma_{21} & \gamma_{20} \\
0 & 0 & 0 & \cdots & 0 & \gamma_{13} & \gamma_{12} & 1 & \gamma_{10} \\
0 & 0 & 0 & 0 & \cdots & 0 & \gamma_{02} & \gamma_{01} & 1
\end{array}\right) \\
& \boldsymbol{Q}=\left(\begin{array}{ccccccccccc}
b_{00} & b_{01} & b_{02} & b_{03} & b_{04} & b_{05} & b_{06} & 0 & 0 & \cdots & 0 \\
b_{10} & b_{11} & b_{12} & b_{13} & b_{14} & b_{15} & b_{16} & 0 & 0 & \cdots & 0 \\
b_{20} & b_{21} & b_{22} & b_{23} & b_{24} & b_{25} & b_{26} & 0 & 0 & \cdots & 0 \\
-a_{3} & -a_{2} & -a_{1} & 0 & a_{1} & a_{2} & a_{3} & 0 & 0 & \cdots & 0 \\
0 & -a_{3} & -a_{2} & -a_{1} & 0 & a_{1} & a_{2} & a_{3} & 0 & \cdots & 0 \\
\vdots & \ddots & \ddots & \ddots & \ddots & \ddots & \ddots & \ddots & \ddots & \ddots & \vdots \\
0 & \cdots & 0 & -a_{3} & -a_{2} & -a_{1} & 0 & a_{1} & a_{2} & a_{3} & 0 \\
0 & \cdots & 0 & 0 & -a_{3} & -a_{2} & -a_{1} & 0 & a_{1} & a_{2} & a_{3} \\
0 & \cdots & 0 & 0 & -b_{26} & -b_{25} & -b_{24} & -b_{23} & -b_{22} & -b_{21} & -b_{20} \\
0 & \cdots & 0 & 0 & -b_{16} & -b_{15} & -b_{14} & -b_{13} & -b_{12} & -b_{11} & -b_{10} \\
0 & \cdots & 0 & 0 & -b_{06} & -b_{05} & -b_{04} & -b_{03} & -b_{02} & -b_{01} & -b_{00}
\end{array}\right)
\end{aligned}
$$

and

$$
\overline{\mathbf{f}}^{\prime}=\left(\bar{f}_{0}^{\prime}, \bar{f}_{1}^{\prime}, \bar{f}_{2}^{\prime}, \cdots, \bar{f}_{N}^{\prime}\right)^{T}, \quad \mathbf{f}=\left(f_{0}, f_{1}, f_{2}, \cdots, f_{N}\right)^{T}
$$

where $\bar{f}_{i}^{\prime}$ is a finite difference approximation to the exact spatial derivative $f_{i}^{\prime}$ at a nodal point $i$ and $b_{i i}=-\sum_{j=0, \neq i}^{6} b_{i j}$. The three boundary closure schemes are applied at the $i=\{0, N\},\{1, N-1\}$ and $\{2, N-2\}$ nodes. They comprise of 27 unique coefficients:

$$
\begin{array}{lll}
\gamma_{i j} \quad \text { for } \quad i=\{0,1,2\} & j=\{0, \cdots, i+2\}, \neq i \\
b_{i j} \quad \text { for } \quad i=\{0,1,2\} & j=\{0, \cdots, 6\}, \neq i .
\end{array}
$$


The central interior scheme consists of five coefficients $\left(\alpha, \beta, a_{1}, a 2, a 3\right)$, and is applied throughout the remainder of the domain $(3 \leq i \leq N-3)$. The template we will consider in the current paper is fourth-order accurate in the interior and at the boundaries. For the interior nodes we implement the optimised fourth-order coefficients suggested by Kim [1]. (Full details of the interior scheme performance, including its modified wavenumber characteristics can be found in [1].)

Fourier series decomposes a dependent function $f$ into a number of oscillatory functions also known as Fourier coefficients $\hat{f}(k)$. For a domain of $N+1$ points $(0, \cdots, N)$ the discrete Fourier series can be expressed as

$$
f(x)=\sum_{k=-N / 2}^{N / 2} \hat{f}(k) \exp \left(\frac{\jmath 2 \pi k x}{L}\right)
$$

where $\jmath=\sqrt{-1}, L$ is the domain length, $x$ is spatial coordinate, and $k$ is the wavenumber. This may be simplified by substituting for a scaled coordinate $x^{*}=x / h$ and a scaled wavenumber $\omega=2 \pi k h / L$, where $h$ is the grid spacing:

$$
f(x)=\sum_{k=-N / 2}^{N / 2} \hat{f}(k) \exp \left(\jmath \omega x^{*}\right) .
$$

After realising that $\left.f_{i \pm m} \equiv f\left(x^{*} \pm m\right)\right)$, where $m \in \mathbb{Z}$, it is possible to derive an expression for a scaled modified wavenumber $\bar{\omega}$ by applying the Fourier transform to each term in a differencing scheme. This differs from the exact wavenumber $\omega$ due to numerical approximation, specifically $f^{\prime}=\jmath \omega f$, while $\bar{f}^{\prime}=\jmath \bar{\omega} f$ (using Eq.(4)).

Dispersion and dissipation errors of differencing schemes are commonly 
quantified using Fourier analysis, applied to each differencing stencil on an individual basis (this procedure is described in detail in [14]). However, this fails to take into account the fact that during actual simulations schemes are not evaluated separately. Rather, they are implemented in a matrix system of equations, as in Eq.(1). By inverting the matrix $\mathbf{P}$, the following expression for the spatial derivative at each nodal point can be obtained:

$$
\overline{\mathbf{f}}^{\prime}=\frac{1}{h} \mathbf{T f}
$$

where $\mathbf{T}=\mathbf{P}^{-1} \mathbf{Q}$. Consequently it seems appropriate to define a modified wavenumber based on the spectral resolution of the whole composite template, by analysing each finite difference stencil in a coupled fashion. This was first suggested by Jordan [12] whose composite template approach consists of taking the Fourier transform of each row of the matrix system in Eq.(1). This results in a pseudo-wavenumber curve for each grid point, with properties dependent on each other point used in the analysis. In the current paper we expand upon this approach by alternatively considering the inverted matrix system given by Eq.(5). Applying the Fourier transform sequentially to each row of this system result in the following:

$$
\left(\begin{array}{c}
j \bar{\omega} \hat{f}(k) \exp \left(j \omega x^{*}\right) \\
j \bar{\omega} \hat{f}(k) \exp \left(j \omega\left(x^{*}+1\right)\right) \\
j \bar{\omega} \hat{f}(k) \exp \left(j \omega\left(x^{*}+2\right)\right) \\
\vdots \\
j \bar{\omega} \hat{f}(k) \exp \left(j \omega\left(x^{*}+N\right)\right)
\end{array}\right)=\mathbf{T}\left(\begin{array}{c}
\hat{f}(k) \exp \left(j \omega x^{*}\right) \\
\hat{f}(k) \exp \left(j \omega\left(x^{*}+1\right)\right) \\
\hat{f}(k) \exp \left(j \omega\left(x^{*}+2\right)\right) \\
\vdots \\
\hat{f}(k) \exp \left(j \omega\left(x^{*}+N\right)\right)
\end{array}\right)
$$


After some algebraic manipulation this leads to an expression for the coupled modified wavenumber of the composite template:

$$
j \overline{\boldsymbol{\omega}}=\left(\begin{array}{c}
T_{00}+T_{01} \exp (j \omega)+T_{02} \exp (2 j \omega)+\cdots+T_{0 N} \exp (N j \omega) \\
T_{10} \exp (-j \omega)+T_{11}+T_{12} \exp (j \omega)+\cdots+T_{1 N} \exp ((N-1) j \omega) \\
\vdots \\
T_{N 0} \exp (-N j \omega)+T_{N 1} \exp (-(N-1) j \omega) \\
+T_{N 2} \exp (-(N-2) j \omega)+\cdots+T_{N N}
\end{array}\right)
$$

Here $\overline{\boldsymbol{\omega}}$ is a vector of length $N+1$, representing the scaled modified wavenumber at each nodal point $(0 \cdots N)$. Each nodal element of $\overline{\boldsymbol{\omega}}$ may also be expressed in a more compact summation form as:

$$
\bar{\omega}_{i}=-j \sum_{m=0}^{N} T_{i m} \exp (j \omega(m-i)) \quad \text { where } i=0,1,2, \cdots, N
$$

where $i$ and $m$ represent the rows and columns of the matrix $\mathbf{T}$. The dispersion and dissipation errors approximated by Eq.(8) should provide a truer depiction of the performance of the finite difference schemes, since the modified wavenumber characteristics are now directly linked to the numerical representation of the derivative.

\section{Boundary Scheme Optimisation Framework}

The chosen optimisation technique for the boundary scheme coefficients is a genetic algorithm (GA) from the Matlab optimisation toolbox. This technique is chosen over more traditional gradient based methods for its ability 
to efficiently and robustly explore nonconvex objective functions and constraints. The Matlab implementation of the GA conveniently handles the selection, crossover and mutation procedures of the optimisation. Additionally it enables simple implementation of nonlinear inequality constraints which prove useful for ensuring numerical stability of the resulting coefficients. For an in-depth discussion of evolutionary optimisation techniques see [15].

\subsection{Formulation of Genetic Algorithm Independent Variables}

The boundary closure schemes are constructed such that they preserve both the truncation error and stencil size of the main interior scheme. Kim [1] achieved this by introducing extrapolation functions based on both trigonometric and fourth-order polynomial series to estimate function values beyond the domain boundaries. By employing a series of constraints for the extrapolation functions to match interior field values and derivatives, the originally central schemes could then be rewritten as a set of non-central differences. The new boundary closure coefficients could then be tuned by use of free control variables left open in the trigonometric series. Similarly, in the current procedure the control variables are selected as the independent variables for the GA, opposed to directly optimising each of the 27 unique boundary closure coefficients. This approach reduces the number of GA independent variables, reducing the complexity of the search task. Furthermore, it ensures the resulting coefficients will obtain the desired fourth-order accuracy. This is specified by the accuracy of the central scheme applied to the boundary nodes and the order of the extrapolation function polynomial series. Originally, Kim [1] proposed an individual extrapolation function for each boundary closure scheme resulting in a total of 11 control variables for optimisation. 
Alternatively, we choose to further simplify the procedure by applying the $i=0$ function to each boundary point $(i=0,1,2)$ resulting in just 3 control variables. The three control variables used for optimisation in the GA are herein referred to as $\phi_{1}, \phi_{2}$ and $\phi_{3}$. Each boundary closure coefficient can be constructed as a non-linear function of these variables. Full details of the extrapolation function and the constraints required to convert $\phi_{1-3}$ into the boundary scheme coefficients can be found in [1].

\subsection{Genetic Algorithm Objective Function}

The purpose of the objective function is to quantify the resolution properties of the composite template so that it may be optimised. Ideally we would like a composite template which produces no dispersion or dissipation errors such that its modified wavenumber curves perfectly match the exact wavenumber:

$$
\begin{aligned}
& \operatorname{Re}(\bar{\omega}) \rightarrow \omega \\
& \operatorname{Im}(\bar{\omega}) \rightarrow 0 .
\end{aligned}
$$

Here $\bar{\omega}$ is determined by Eq.(8) and $\omega$ is the exact scaled wavenumber. We

can represent this requirement as an integral error measure $\mathscr{E}_{i} A$ which we require to tend to zero:

$$
\left.\mathscr{E}_{i}^{A}\right|_{0} ^{r}=\left\{\int_{0}^{r}\left[\operatorname{Re}\left(\bar{\omega}_{i}-\omega\right)+\operatorname{Im}(\bar{\omega})\right]^{2} d \omega\right\}^{\frac{1}{2}} \text { for } i=\{0,1,2\}
$$

An appropriate value for the integration range $(r)$ is obtained by utilising Eq.(11) as an initial objective function in the GA. A bisection type 
trial and error is then conducted to determine which $r$ value produces the most successful optimisation output. This is judged by the performance the newly optimised schemes attain in the one-dimensional scalar wave benchmark problem (section 5.1). This process resulted in a value of $r=0.52 \pi$. Ideally we would like the modified wavenumber to match the exact wavenumber over the full range of scales from $0-\pi$, and would therefore select $r$ to reflect this. However, this tends to result in very large overshoots at higher wavenumbers. Additionally due to the dependence between each schemes coupled modified wavenumber curves, a high integration range often results in the improvement of one schemes resolution characteristics at the expense of another. One possible solution would be to remove high frequencies with filtering operations, however the low cut-off frequency required would greatly degrade the solution obtained. Alternatively, by selecting a more moderate $r$, the characteristics of each scheme tend to remain comparable over at least a intermediate wavenumber range, resulting in far superior aggregate resolution characteristics. The consequence of ignoring the higher wavenumbers in the optimisation is a lack of control over wavenumber characteristics at high frequencies. Despite this, the $i=1$ and $i=2$ schemes remained relatively well behaved. The $i=0$ scheme on the other hand was found to exhibit a fairly large overshoot relative to the exact wavenumber between roughly $0.8 \pi \leq \omega \leq \pi$, albeit reduced compared to an $r=\pi$ case. To compensate for this, an additional error measure $\mathscr{E}^{B}$ is included, which aims to damp the $i=0$ overshoots over the appropriate range.

$$
\left.\mathscr{E}^{B}\right|_{0.8 \pi} ^{\pi}=\left\{\int_{0.8 \pi}^{\pi}\left[\operatorname{Re}\left(\bar{\omega}_{0}-\omega\right)+\operatorname{Im}(\bar{\omega})\right]^{2} d \omega\right\}^{\frac{1}{2}}
$$


Combining $\mathscr{E}^{A}$ and $\mathscr{E}^{B}$ results in a final blended error measure $\mathscr{E}$ used as the objective function in the GA

$$
\mathscr{E}=\eta\left(\left.\sum_{i=0}^{2} \mathscr{E}_{i}^{A}\right|_{0} ^{0.52 \pi}\right)+\left.(1-\eta) \mathscr{E} B\right|_{0.8 \pi} ^{\pi}
$$

where $\eta$ is the weighting factor between the two error measures. If $\eta$ is too large, the overshoots at $i=0$ are not sufficiently damped, however if $\eta$ is too small the aggregate resolution characteristics of the composite template begin to suffer. A suitable value for $\eta$ was selected through an equivalent trial and error procedure as outlined for the integration range $r$, eventually yielding a value of $\eta=0.948$.

\subsection{Linear Stability Constraint}

In addition to exhibiting good resolution properties, and maintaining a desired order of accuracy, a successful numerical scheme must also consistently provide stable solutions. In past procedures the numerical stability is often treated as an afterthought, and is only considered after the wavenumber optimisation of the finite difference coefficients is completed $([1,11-13])$. This usually results in the use of trial and error routines to obtain a result which both achieves good performance and meets the desired stability criteria. In the current work a more consistent optimisation strategy is established by integrating a non-linear constraint for eigenvalue stability into the GA, ensuring the optimisation output is always satisfactory.

As pointed out by Liu et al. [11] and Carpenter et al. [10], the optimisation process can often be detrimental to numerical stability, limiting the number of feasible solutions. For this reason the authors suggests that the finite 
difference schemes are used in conjunction with a stabilising technique. In this case we use the 6 th order compact filters provided by Kim [16]. Compact filters improve numerical stability by introducing a cut-off frequency. This effectively removes unresolved wavenumber components from the solution at the end of each time step. They are including via the following modifications to Eq.(1):

$$
\mathbf{P} \overline{\mathbf{f}}^{\prime}=\frac{1}{h} \mathbf{Q}(\mathbf{f}+\tilde{\Delta} \mathbf{f})
$$

and

$$
\tilde{\Delta} \mathbf{f}=\left(\tilde{\Delta} f_{0}, \tilde{\Delta} f_{1}, \tilde{\Delta} f_{2}, \cdots, \tilde{\Delta} f_{N}\right)^{T}
$$

where $\tilde{\Delta} f_{i}=\tilde{f}_{i}-f_{i}$ represents the difference between filtered and unfiltered values. A solution for $\tilde{\Delta} \mathbf{f}$ can be obtained by solving the following matrix system of equations

$$
\mathbf{R} \tilde{\Delta} \mathbf{f}=\mathbf{S f}
$$

where

$$
\boldsymbol{R}=\left(\begin{array}{ccccccccc}
1 & \gamma_{01}^{F} & \gamma_{02}^{F} & 0 & \cdots & 0 & 0 & 0 & 0 \\
\gamma_{10}^{F} & 1 & \gamma_{12}^{F} & \gamma_{13}^{F} & 0 & \cdots & 0 & 0 & 0 \\
\gamma_{20}^{F} & \gamma_{21}^{F} & 1 & \gamma_{23}^{F} & \gamma_{24}^{F} & 0 & \cdots & 0 & 0 \\
0 & \beta^{F} & \alpha^{F} & 1 & \alpha^{F} & \beta^{F} & 0 & \cdots & 0 \\
\vdots & \ddots & \ddots & \ddots & \ddots & \ddots & \ddots & \ddots & \vdots \\
0 & \cdots & 0 & \beta^{F} & \alpha^{F} & 1 & \alpha^{F} & \beta^{F} & 0 \\
0 & 0 & \cdots & 0 & \gamma_{24}^{F} & \gamma_{23}^{F} & 1 & \gamma_{21}^{F} & \gamma_{20}^{F} \\
0 & 0 & 0 & \cdots & 0 & \gamma_{13}^{F} & \gamma_{12}^{F} & 1 & \gamma_{10}^{F} \\
0 & 0 & 0 & 0 & \cdots & 0 & \gamma_{02}^{F} & \gamma_{01}^{F} & 1
\end{array}\right)
$$




$$
\boldsymbol{S}=\left(\begin{array}{ccccccccccc}
0 & 0 & 0 & 0 & 0 & 0 & 0 & 0 & 0 & \cdots & 0 \\
0 & 0 & 0 & 0 & 0 & 0 & 0 & 0 & 0 & \cdots & 0 \\
b_{20}^{F} & b_{21}^{F} & b_{22}^{F} & b_{23}^{F} & b_{24}^{F} & b_{25}^{F} & 0 & 0 & 0 & \cdots & 0 \\
a_{3}^{F} & a_{2}^{F} & a_{1}^{F} & a_{0}^{F} & a_{1}^{F} & a_{2}^{F} & a_{3}^{F} & 0 & 0 & \cdots & 0 \\
0 & a_{3}^{F} & a_{2}^{F} & a_{1}^{F} & a_{0}^{F} & a_{1}^{F} & a_{2}^{F} & a_{3}^{F} & 0 & \cdots & 0 \\
\vdots & \ddots & \ddots & \ddots & \ddots & \ddots & \ddots & \ddots & \ddots & \ddots & \vdots \\
0 & \cdots & 0 & a_{3}^{F} & a_{2}^{F} & a_{1}^{F} & a_{0}^{F} & a_{1}^{F} & a_{2}^{F} & a_{3}^{F} & 0 \\
0 & \cdots & 0 & 0 & a_{3}^{F} & a_{2}^{F} & a_{1}^{F} & a_{0}^{F} & a_{1}^{F} & a_{2}^{F} & a_{3}^{F} \\
0 & \cdots & 0 & 0 & 0 & b_{25}^{F} & b_{24}^{F} & b_{23}^{F} & b_{22}^{F} & b_{21}^{F} & b_{20}^{F} \\
0 & \cdots & 0 & 0 & 0 & 0 & 0 & 0 & 0 & 0 & 0 \\
0 & \cdots & 0 & 0 & 0 & 0 & 0 & 0 & 0 & 0 & 0
\end{array}\right)
$$

superscript $F$ denotes a filter coefficient, $a_{0}^{F}=-2\left(a_{1}^{F}+a_{2}^{F}+a_{3}^{F}\right)$, and $b_{22}^{F}=-\left(b_{20}^{F}+b_{21}^{F}+b_{23}^{F}+b_{24}^{F}+b_{25}^{F}\right)$.

The filter coefficients for each point are calculated by a global cut-off frequency $\Omega^{c}$, and individual boundary weighting factors $w_{i}$ (for the exact relations see [16]). Cut-off frequencies for each point are calculated as follows:

$$
\Omega_{i}^{c}=\left\{\begin{array}{lll}
\Omega^{c} & \text { for } & i \in[3, \ldots, N-3], \\
\left(1-w_{2}\right) \Omega^{c} & \text { for } & i=\{2, N-2\}, \\
\left(1-w_{1}\right) \Omega^{c} & \text { for } \quad i=\{1, N-1\}, \\
\left(1-w_{0}\right) \Omega^{c} & \text { for } \quad i=\{0, N\},
\end{array}\right.
$$

For his boundary finite difference schemes Kim [16] suggested weighting factors of $w_{0} / 3=w_{1} / 2=w_{2}=0.085$. In the current approach the same linear relationship is maintained with $w_{2}$ implemented as a fourth independent variable $\left(\phi_{4}\right)$ in the GA. This allows us to determine optimal boundary weighting factors for the new template when a given cut-off frequency is used, in this case $\Omega^{c}=0.88 \pi$.

Stability of a finite-difference systems is usually verified through eigen- 
value analysis of a 1D linear scalar wave problem, identified by the following equation

$$
\frac{\partial f}{\partial t}+c_{\infty} \frac{\partial f}{\partial x}=0
$$

where $c_{\infty}$ is the wave convection speed. The domain spans from $x \in[0, L]$, and is discretised into $N+1$ points, with the only boundary condition $f(x=0, t)=0$, applied at the inlet. The spatial derivative $(\partial f / \partial x)$ can be numerically approximated by substituting Eq.(14) and (16) into Eq.(18), resulting in the following system of ODEs [16]:

$$
\mathbf{P} \frac{d \mathbf{f}}{d t}=-\frac{c_{\infty}}{h} \mathbf{Q}\left(\mathbf{I}+\mathbf{R}^{-1} \mathbf{S}\right) \mathbf{f}
$$

The solution to this problem is simply $\mathbf{f}=\mathbf{v} \exp (a t)$ where $a$ controls the decay/growth rate. Substituting for $\mathbf{f}$ in Eq.(19) yields

$$
\mathbf{Q}\left(\mathbf{I}+\mathbf{R}^{-1} \mathbf{S}\right) \mathbf{v}=-\lambda \mathbf{P} \mathbf{v}
$$

where $\lambda=a h / c_{\infty}$ represent the eigenvalues and $\mathbf{v}$ the eigenvectors of the system. For stability it is required that $a \leq 0$ such that $\operatorname{Re}\left(\lambda_{\max }\right) \leq 0$. Using a Heaviside step function this could be implemented as a non-linear constraint in the GA by: $H\left(\operatorname{Re}\left(\lambda_{\max }\right)\right)-1 / 2 \leq 0$. However the issue with this formulation is a discontinuity close to zero. To resolve this we can employ the following relation

$$
H(x)=\frac{1}{2} \lim _{s \rightarrow \infty}(1+\tanh (s x))
$$

where $s$ determines the gradient near zero. When $s=1$ this leads to the 
following continuous non-linear constraint:

$$
\frac{1}{2} \tanh \left(\operatorname{Re}\left(\lambda_{\max }\right)\right) \leq 0
$$

A solution generated by the GA is only considered feasible if it satisfies this constraint over 3 grid levels, $N=50,100$ and 200 .

\section{Optimisation Output}

The first step in the optimisation process is to randomly generate a population of 50 chromosomes (potential solutions) which satisfy the optimisation constraints in the GA. Each individual contains four genes, three representing potential values for the control variables $\left(\phi_{1}, \phi_{2}, \phi_{3}\right)$ and one representing a value for the boundary weighting factor $\left(w_{2}=\phi_{4}\right)$. Each chromosome is then ranked according to its fitness score produced by the fitness/objective function. Pairs of chromosomes are selected by a stochastic uniform strategy for reproduction. Eighty percent of new solutions are generated via a scattered crossover function assigning genes from the two parents based on a random binary vector. For example a bit string of [110] means the first and second genes should be inherited from the first parent, and the third gene from the second. The further twenty percent of new solutions are created by random mutation. Finally the two most promising individuals are guaranteed to progress to the next iteration. This process is continued until the average weighted change in the objective function falls below a certain tolerance, set to $10^{-6}$. The resultant values for $\phi_{1}-\phi_{4}$ can be found in Table 1 , and the corresponding boundary scheme coefficients in Table 2. 


\begin{tabular}{cccc}
\hline$\phi_{1}$ & $\phi_{2}$ & $\phi_{3}$ & $\phi_{4}$ \\
\hline 0.3319 & 0.1932 & 1.7329 & 0.0485 \\
\hline
\end{tabular}

Table 1: Optimisation output

\begin{tabular}{cccc}
\hline Coefficient & $i=0$ & $i=1$ & $i=2$ \\
\hline$\gamma_{i 0}$ & - & 0.11737546726594537 & -0.067477420334188354 \\
$\gamma_{i 1}$ & 9.2793108237360826 & - & -0.1945509344676567 \\
$\gamma_{i 2}$ & 9.8711877434133051 & 0.92895849448052303 & - \\
$\gamma_{i 3}$ & - & -0.067839996199150834 & 1.279565347145571 \\
$\gamma_{i 4}$ & - & - & 0.20842348769505742 \\
$b_{i 0}$ & - & -0.4197688256685424 & 0.20875393530974462 \\
$b_{i 1}$ & -9.9196421679170452 & - & -0.36722447739446801 \\
$b_{i 2}$ & 10.088151775649886 & 1.1593253854830003 & - \\
$b_{i 3}$ & 4.1769460418803268 & 0.31685797023808876 & 0.98917602108458036 \\
$b_{i 4}$ & -0.82222305192207212 & -0.096453054902842381 & 0.63518969715000262 \\
$b_{i 5}$ & 0.14757709267988142 & 0.015579947274307879 & 0.0042145635666246068 \\
$b_{i 6}$ & -0.014332365879513103 & -0.0014553614585464077 & 0.0010111910030585999 \\
\hline
\end{tabular}

Table 2: Optimised boundary coefficients appearing in section 2.

\subsection{Composite Template Modified Wavenumber Characteristics}

The coupled modified wavenumber properties of a composite template are dependent on the number of grid points we choose to analyse. To precisely represent its resolution characteristics we would need to include the effect of every grid point we plan to use in our simulation. Clearly this becomes impractical for simulations of any meaningful size due to cost constraints. For this reason optimisations are based on the simplest scenario, a 7 by 7 matrix system consisting of 3 boundary schemes on either side of the domain and 1 central interior point. Additionally since the modified wavenumber characteristics of the template will be symmetrical about the centre point we will only analyse the first 4 points. Although this is the most fundamental system we can examine it is found to be more than sufficient at demonstrating the effectiveness of the new optimisation approach, with the resulting schemes 
producing substantial accuracy gains in section 5 .
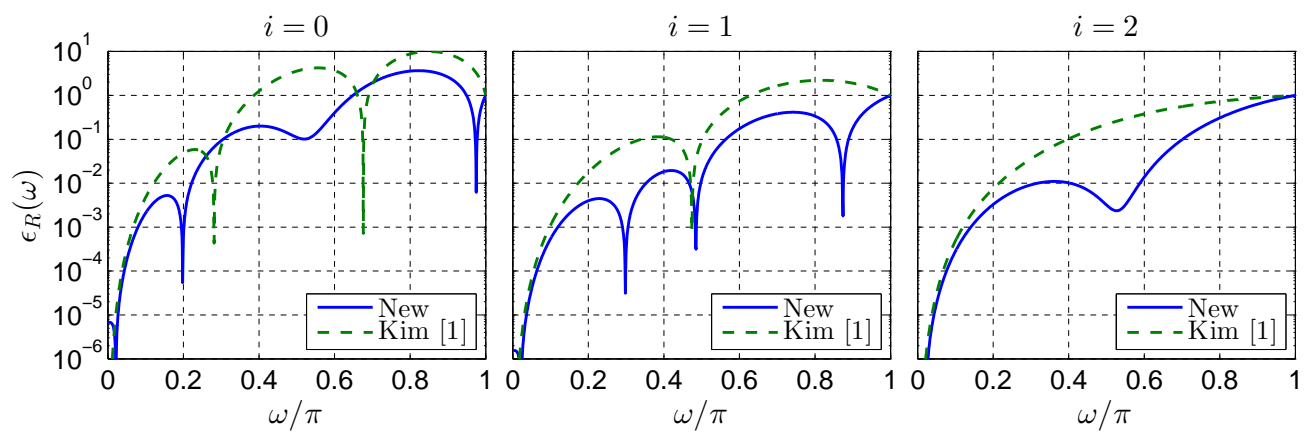

Figure 1: Real wavenumber errors produced by the the new finite difference template and that of Kim [1] at the three boundary nodes $(i=0,1,2)$ based on a $N=7$ matrix system
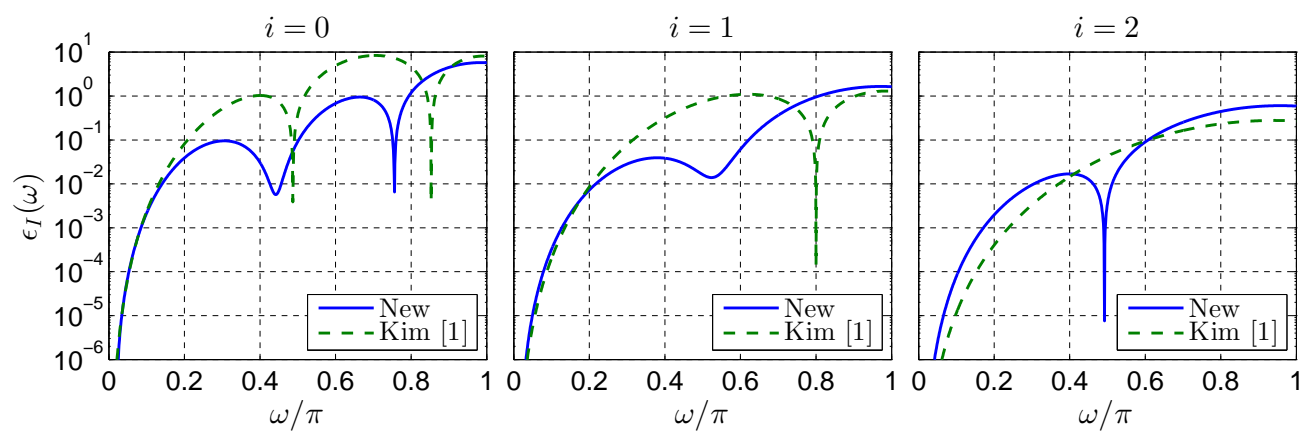

Figure 2: Imaginary wavenumber errors produced by the the new finite difference template and that of $\operatorname{Kim}[1]$ at the three boundary nodes $(i=0,1,2)$ based on a $N=7$ matrix system

Since the resolution properties of each point in Eq.(8) are coupled, it is possible that optimising one point in the composite template can have a detrimental effect on others. For this reason resolution errors tend to be higher than if each scheme were analysed individually. This makes it very hard to draw comparisons between resolution errors obtained in studies based on a decoupled approach. Consequently comparisons are made with the schemes provided by Kim [1], based solely on the new approach. Figures 1 

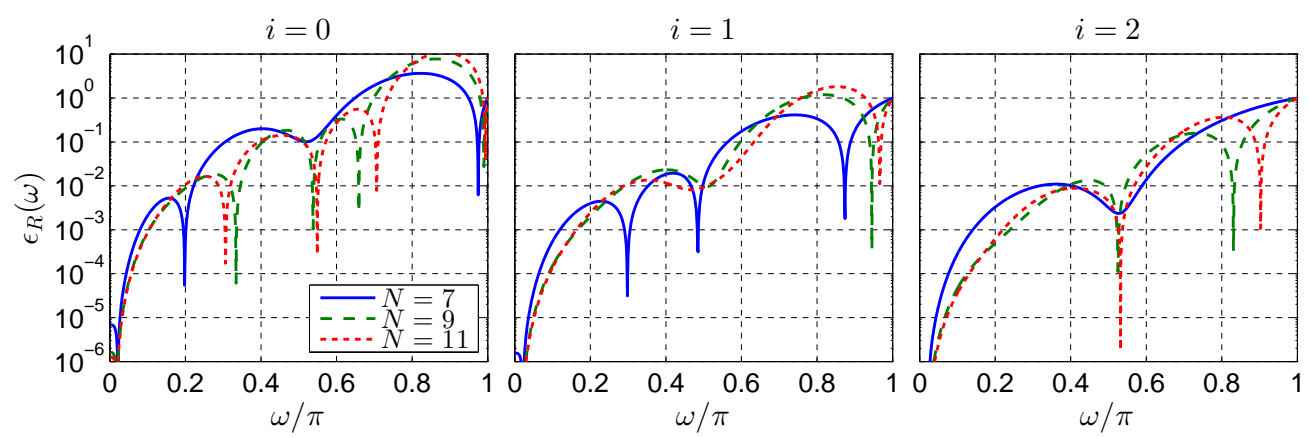

Figure 3: Real wavenumber errors produced by the the new finite difference template at the three boundary nodes $(i=0,1,2)$ with an increasing number of points analysed $(N=7,9,11)$
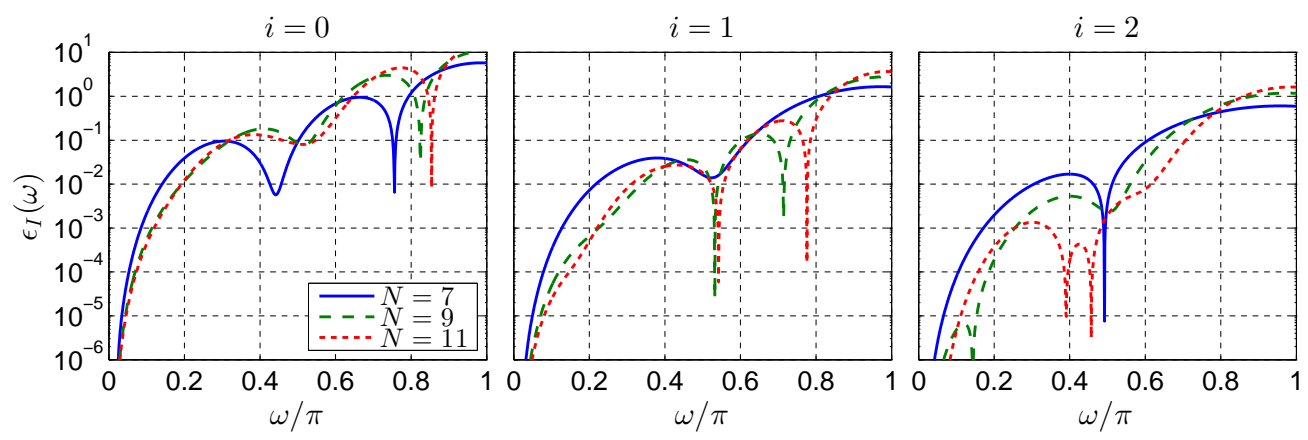

Figure 4: Imaginary wavenumber errors produced by the the new finite difference template at the three boundary nodes $(i=0,1,2)$ with an increasing number of points analysed $(N=7,9,11)$
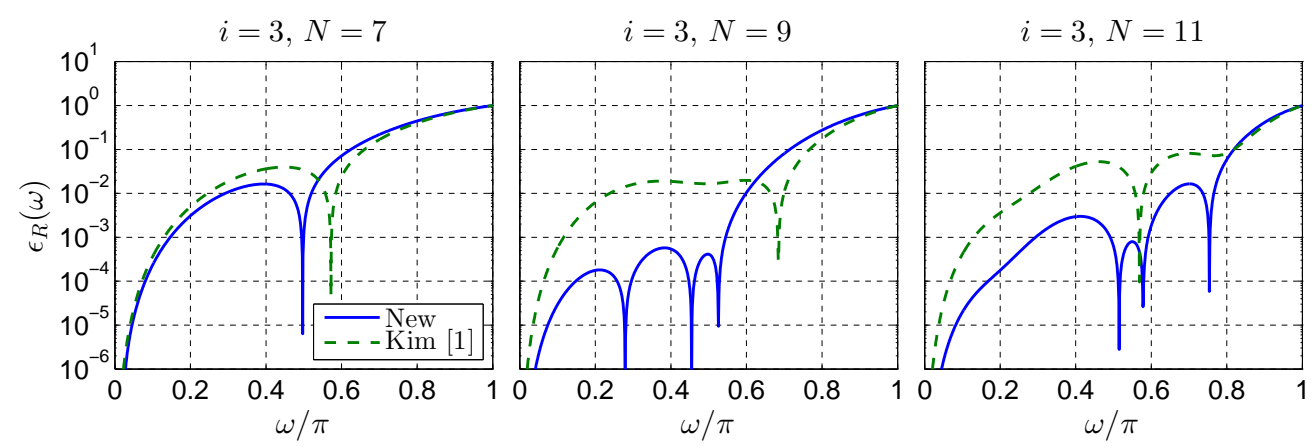

Figure 5: Real wavenumber error produced by the the new finite difference template and that of Kim [1] at the first interior node $(i=3)$ with an increasing number of points analysed $(N=7,9,11)$ 

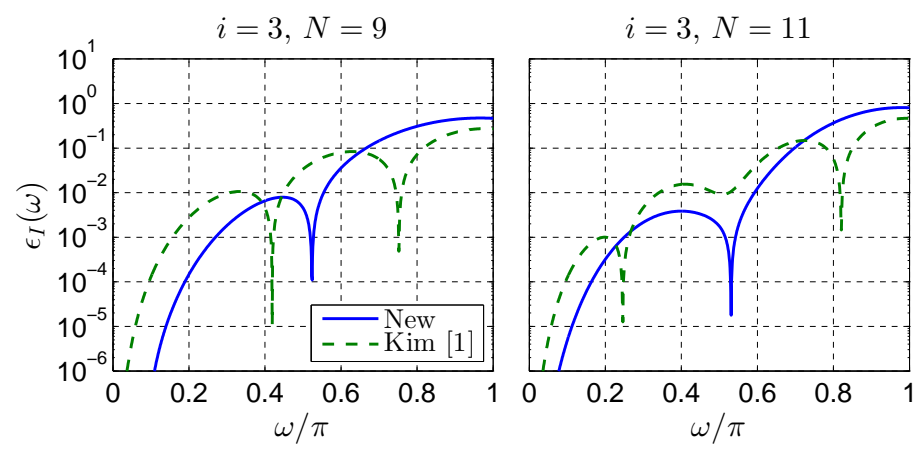

Figure 6: Imaginary wavenumber error produced by the the new finite difference template and that of $\operatorname{Kim}[1]$ at the first interior node $(i=3)$ with $N=9$ and $N=11 . N=7$ is not shown as the dissipation error is zero due to the $i=3$ being located at the centre of the composite template.

and 2 describe the respective dispersion and dissipation properties produced by Kim's template and the current study. Differences between $\omega$ and $\bar{\omega}$ at each nodal point $i$ are measured by means of a relative error for both real and imaginary components [1]:

$$
\begin{gathered}
\epsilon_{R, i}(\omega)=\left|\frac{\operatorname{Re}\left(\bar{\omega}_{i}\right)-\omega}{\omega}\right| \\
\epsilon_{I, i}(\omega)=\left|\frac{\operatorname{Im}\left(\bar{\omega}_{i}\right)}{\omega}\right|
\end{gathered}
$$

The wavenumber range for which the dispersion and dissipation errors are below a specified tolerance $\sigma$, can be identified by the critical wavenumbers $\omega_{R c, i}^{\sigma}$ and $\omega_{I c, i}^{\sigma}$, such that $\epsilon_{R, i}<\sigma$ for $0 \leq \omega \leq \omega_{R c, i}^{\sigma}$ and $\epsilon_{I, i}<\sigma$ for $0 \leq \omega \leq$ $\omega_{I c, i}^{\sigma}$, with $0 \leq \omega \leq \pi$. Table 3 shows a comparison of the critical wavenumbers attained using $\sigma=0.01,0.05$ and 0.1 . Overall the newly optimised template offers greatly reduced resolution errors. The only exception is the dissipation error of the $i=2$ scheme, which is increased outside of the range 
$0.41 \leq \omega \leq 0.61$. This occurs because the objective function (Eq.(13)) aims to reduce the resolution error of the composite template as a whole. In the case of the $i=2$ scheme this results is some increase to the dissipation error relative to Kim's scheme [1], but a more substantial reduction to dispersion error.

Figures 3 and 4 show the influence of the number of grid points $(N)$ used in the modified wavenumber analysis on the boundary scheme dispersion and dissipation errors. Each matrix system implements 6 boundary nodes, and an increasing number of interior nodes $(N-6)$. The wavenumber errors quickly converge as $N$ is increased, with only small changes observed between the $N=7$ and $N=9,11$ cases.

Although the coefficients of the first interior point is fixed its resolution properties will be altered by the adjacent boundary schemes due to the fully coupled nature of the modified wavenumber formulation. Figure 5 shows the dispersion error of the new template and that of Kim [1] obtained at the $i=3$ node with increasing values of $N$. For $N=7$ the new template offers some improvement to the critical wavenumber based on the stricter $\sigma=0.01$ error tolerance. As $N$ increases a much larger improvement is revealed. At $N=11$ the new schemes achieve a critical wavenumber of $\omega_{R c, 3}^{0.01}=0.651 \pi$ compared to $0.277 \pi$ for those of Kim [1]. This highlights how the reductions made to the resolution error at the boundaries has a positive knock-on effect at the near boundary interior nodes. The dissipation errors for the $i=3$ node are compared in Figure 6 for $N=9$ and $N=11$. $(N=7$ is not included in this case as its dissipation error is zero due to the $i=3$ node being located at the centre of the composite template.) Similar to the dispersion errors, 
the new template obtains an improved critical wavenumber of $\omega_{I c, 3}^{0.01}=0.593 \pi$ compared to $0.341 \pi$ for the template of Kim [1].

\begin{tabular}{ccccccc}
\hline \multicolumn{3}{c}{ New schemes } & \multicolumn{3}{c}{ Kim [1] } \\
\hline & $\sigma=0.01$ & $\sigma=0.05$ & $\sigma=0.1$ & $\sigma=0.01$ & $\sigma=0.05$ & $\sigma=0.1$ \\
\hline$\omega_{R c, 0}^{\sigma}$ & $0.223 \pi$ & $0.269 \pi$ & $0.306 \pi$ & $0.123 \pi$ & $0.203 \pi$ & $0.308 \pi$ \\
$\omega_{I c, 0}^{\sigma}$ & $0.142 \pi$ & $0.217 \pi$ & $0.504 \pi$ & $0.134 \pi$ & $0.183 \pi$ & $0.210 \pi$ \\
$\omega_{R c, 1}^{\sigma}$ & $0.354 \pi$ & $0.534 \pi$ & $0.565 \pi$ & $0.174 \pi$ & $0.267 \pi$ & $0.342 \pi$ \\
$\omega_{I c, 1}^{\sigma}$ & $0.219 \pi$ & $0.593 \pi$ & $0.623 \pi$ & $0.207 \pi$ & $0.278 \pi$ & $0.317 \pi$ \\
$\omega_{R c, 2}^{\sigma}$ & $0.319 \pi$ & $0.660 \pi$ & $0.703 \pi$ & $0.217 \pi$ & $0.329 \pi$ & $0.398 \pi$ \\
$\omega_{I c, 2}^{\sigma}$ & $0.307 \pi$ & $0.566 \pi$ & $0.609 \pi$ & $0.373 \pi$ & $0.518 \pi$ & $0.610 \pi$ \\
$\omega_{R c, 3}^{\sigma}$ & $0.293 \pi$ & $0.579 \pi$ & $0.626 \pi$ & $0.247 \pi$ & $0.626 \pi$ & $0.662 \pi$ \\
\hline
\end{tabular}

Table 3: Critical wavenumbers obtained by the the new template and that of Kim [1] based on an $N=7$ matrix system at the $i=0,1,2$ and 3 nodes utilising various tolerances $(\sigma)$

\subsection{Stability Analysis}

As shown in Table 1 the outcome of the GA stability constraint for the filter cut-off was the boundary weighting factor $w_{2}=0.0485$. The eigenvalue distribution for these settings is shown in Figure 7. As desired the real parts of all eigenvalues have been restricted into the left half plane. The optimised boundary weighting obtains stable eigenvalues over the filter cut-off range $0.74 \pi \leq \Omega_{c} \leq 0.88 \pi$, despite the constraint focusing only on the upper stability limit $\Omega_{c}=0.88 \pi$. If a lower cut-off value is desired a stable solution can still be obtained by reverting to the default value $w_{2}=0.085$. In order to obtain the largest magnitude negative real eigenvalues over the longest stability range the authors suggest implementing the following strategy for $w_{2}$ 


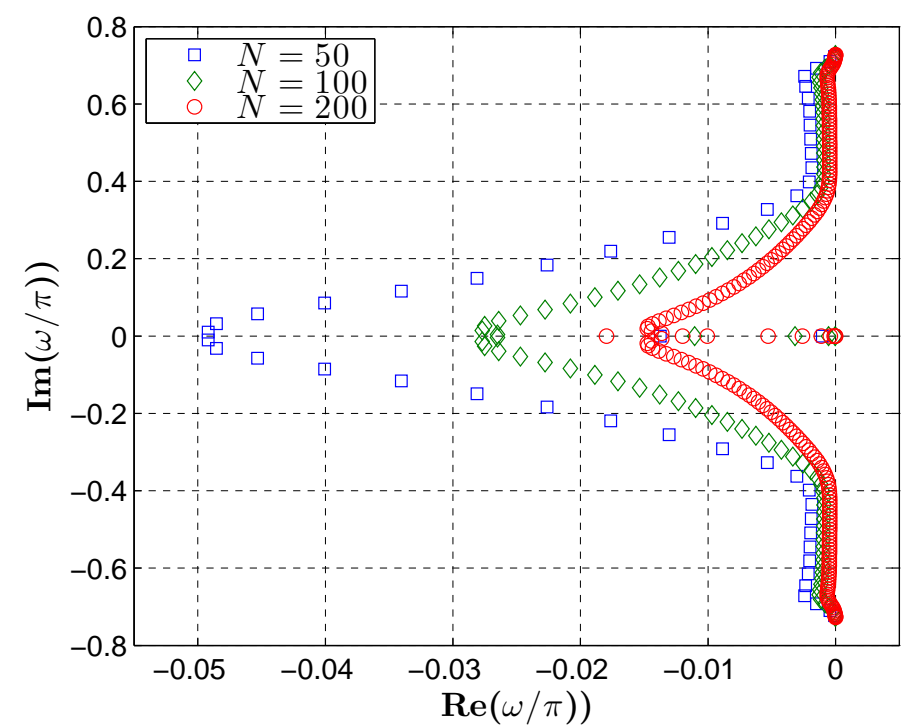

Figure 7: Eigenvalues at various grid sizes for the newly optimised finite difference template with compact filtering

$$
w_{2}= \begin{cases}0.0850 & \text { for } \quad 0.59 \pi \leq \Omega_{c}<0.86 \pi \\ 0.0485 & \text { for } \quad 0.86 \pi \leq \Omega_{c} \leq 0.88 \pi\end{cases}
$$

By adopting this strategy a stable eigenvalue distribution is attainable over the filter cut-off range $0.59 \pi \leq \Omega_{c} \leq 0.88 \pi$. Figure 8 shows the maximum real eigenvalues obtained over this range utilising both weighting factors accordingly.

A more extensive stability analysis can be preformed through application of a test function to a scalar linear wave convection problem, again described by Eq.(18). In this particular instance a modulated wave described by the following initial and boundary conditions is considered: 


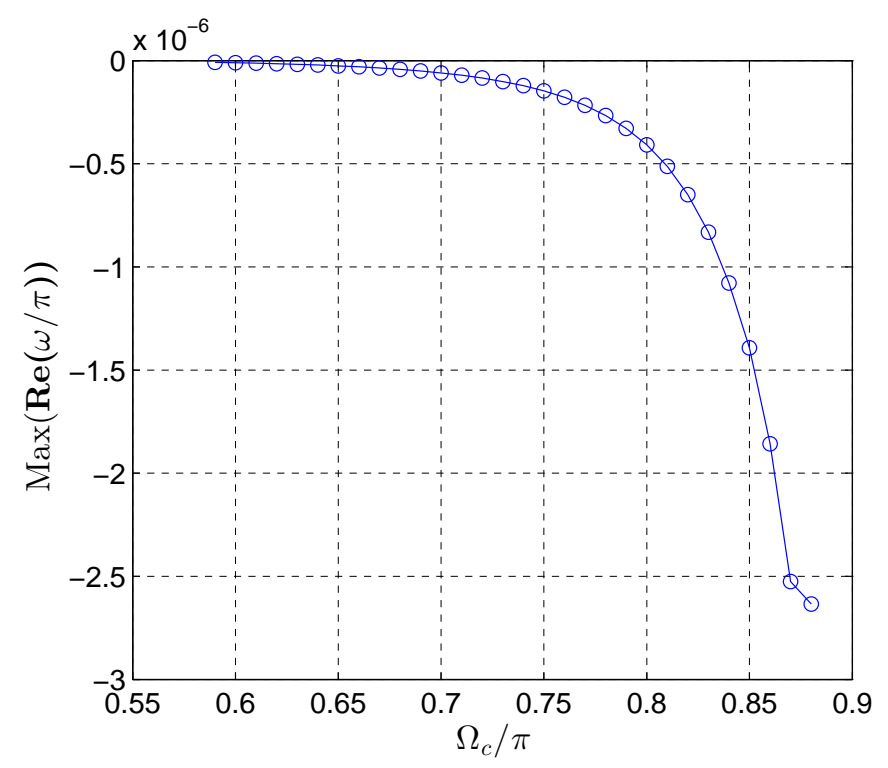

Figure 8: Maximum real eigenvalues for the new schemes with compact filtering over the stable range $0.59 \pi \leq \Omega_{c} \leq 0.88 \pi$. $\left(w_{2}=0.0485\right.$ for $0.86 \pi \leq \Omega_{c} \leq 0.88 \pi$ and $w_{2}=0.085$ for $\left.0.59 \pi \leq \Omega_{c}<0.85 \pi\right) N=200$

$$
\begin{gathered}
f(x, t=0)=f_{\infty}\left[1+A \cos \left(\frac{k_{1} x}{L}\right)\right] \sin \left(\frac{k_{2} x}{L}\right), \\
f(x=0, t)=f_{\infty}\left[1+A \cos \left(\frac{-c_{\infty} k_{1} t}{L}\right)\right] \sin \left(\frac{-c_{\infty} k_{2} t}{L}\right) .
\end{gathered}
$$

Here the frequency and amplitude of the carrier wave component are represented by $k_{2}=25 k_{1}$ and $f_{\infty}$ respectively. Equivalently, $k_{1}=2 \pi$ and $A=1.5$ represent the frequency and amplitude of the modulating component. The boundary schemes are implemented at both the inlet and outlet to the domain. To obtain the exact solution to this problem $x$ is substituted for $\hat{x}=x-c_{\infty} t$ in Eq.(26). Stability of the new template and filters is tested by monitoring errors produced in the simulation for an extended duration. For 


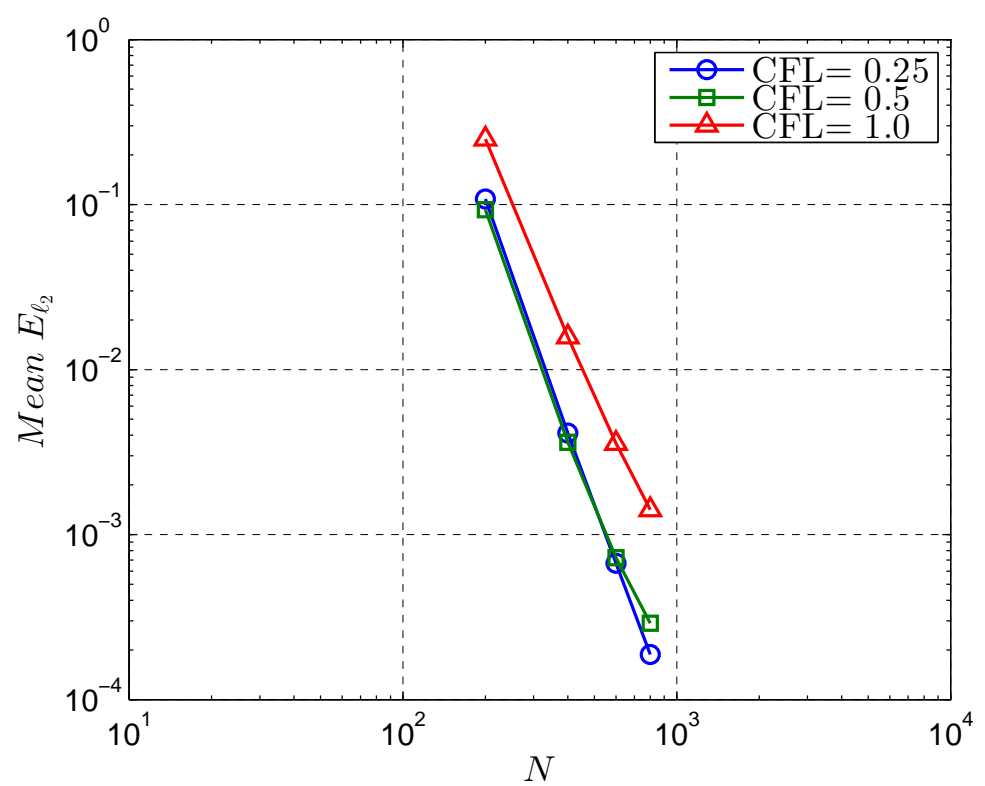

Figure 9: Mean $\ell_{2}$-norm errors produced by the current schemes in calculation of a modulated linear wave for $\mathrm{CFL}=0.25, \mathrm{CFL}=0.5$ and $\mathrm{CFL}=1.0$

coarser grid sizes the calculation is continued until $t=150 \mathrm{~L} / \mathrm{c}_{\infty}$, while for the finest grid $(N=800)$ it is run until $t=10 L / c_{\infty}$ to minimise computational cost. Temporal discretisation is achieved with classical 4th order RungeKutta. To quantify numerical errors the following $\ell_{2}$-norm error is defined:

$$
E_{\ell_{2}}=\left\{\sum_{i=1}^{N}\left[f_{i}-f_{\text {exact }}\right]^{2} /\left(N f_{\infty}^{2}\right)\right\}^{\frac{1}{2}}
$$

Figure 9 shows the time averaged $\ell_{2}$-norm errors produced during calculation of the linear modulated wave for various CFL numbers. Stable solutions with a high-order accurate convergence rate are achieved for all cases. 


\subsection{Comparison to Classical Methods}

The accuracy enhancements made available by utilising pentadiagonal compact schemes including the newly optimised boundaries is shown in Figure 10. It displays the $\ell_{2}$-norm error time history (Eq.(28)) obtained during calculation of a 1D linear scalar wave convection problem utilising different spatial discretisation schemes and $N=400$ grid cells. Temporal discretisation is conducted with classical 4th order Runge-Kutta with a value of 0.5 for the Courant-Friedrichs-Lewy condition (CFL). A full description of this problem is given in section 5.1. The classical explicit method (4th order central interior and 3rd order boundaries) is capable of obtaining a stable solution, albeit with a very large peak error. This highlights the requirement for grid refinement in order to obtain a more acceptable accuracy, inevitably increasing the computational cost. Adopting an implicit method can be an effective way to reduce the level of error for a given grid spacing. This is apparent over the region $t c_{\infty} / L<0.4$, where the standard 4 th order tridiagonal Padé scheme, used here with 2nd order implicit boundaries [17] achieves notably better performance. However such methods often suffer from stability issues, as shown by the divergence at a later time step. This demonstrates the requirement for scheme optimisation to achieve higher levels of accuracy and computational efficiency, without neglecting numerical stability. In the case of the current pentadiagonal system, error reductions in excess of two orders of magnitude are achieved relative to the explicit method, while still maintaining a stable solution. 


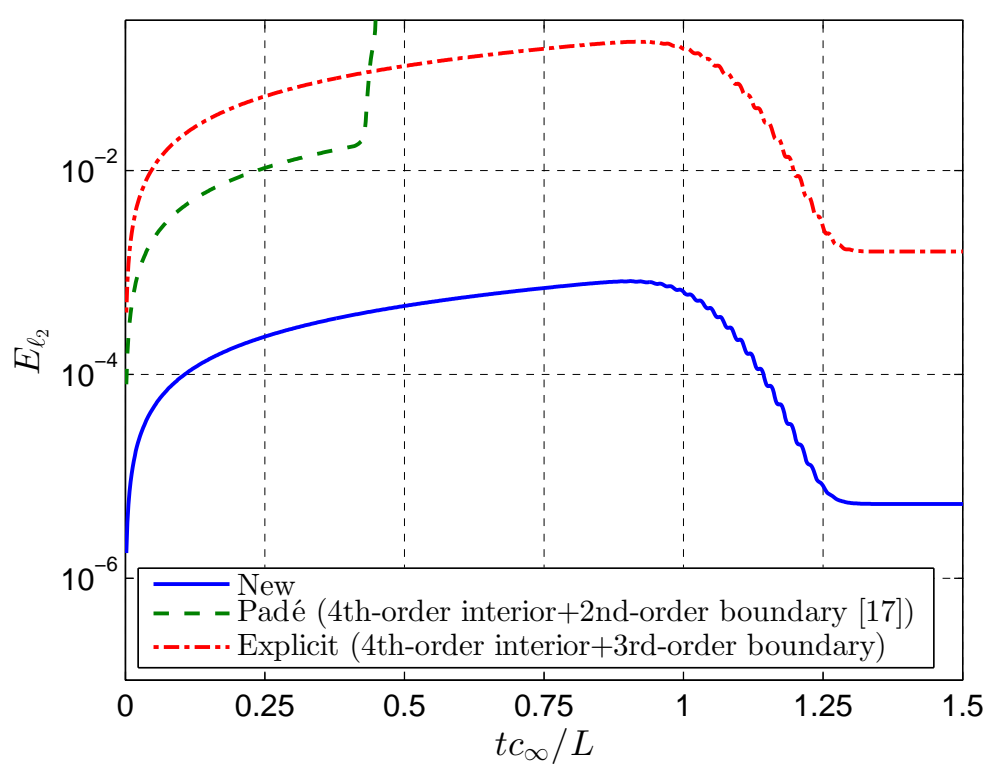

Figure 10: Comparison of $\ell_{2}$-norm error time histories obtained during the $1 \mathrm{D}$ scalar wave convection problem described in section 5.1. Results shown for a classical 4th order explicit finite difference scheme with 3rd order explicit boundaries, a classical 4th order Padé scheme with 2nd order implicit boundaries [17], and the current numerical setup. The simulation is conducted with $N=400$ grid cells, using CFL=0.5 with Runge-Kutta as the time stepping algorithm 


\section{Benchmark Problems}

\subsection{One-dimensional Scalar Wave}

The first benchmark problem we consider is the convection of a onedimensional scalar wave. This problem was first proposed by Tam [18] at the Fourth Computational Aeroacoustics Workshop on Benchmark Problems. It consists of the simulation of a wave pulse as it travels from its initial location within the domain through a computational exit boundary. Unlike the wave convection problem used to analyse the long term linear stability of the finite difference schemes in section 4 , the wave in this problem will entirely leave the domain, resulting in a final solution of zero. This allows us to analyse the capability of the proposed schemes at minimising error reflections at computational boundaries. The initial wave pulse is defined as

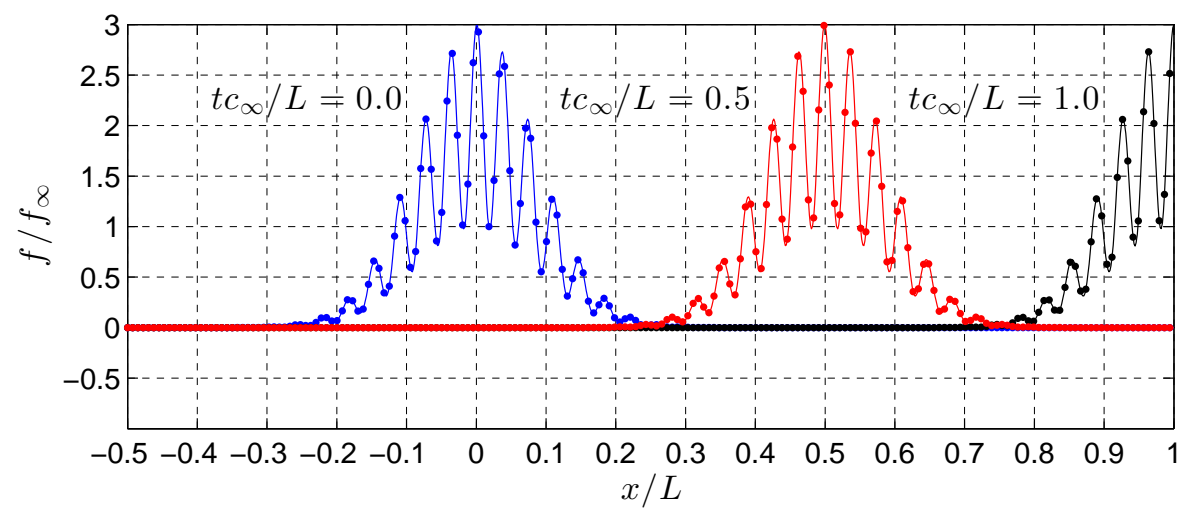

Figure 11: 1D scalar wave at three instances of time $-=$ exact solution, $\bullet=$ numerical solution for $N=1000$ and $\mathrm{CFL}=0.5$

$$
f(x, t=0)=f_{\infty}\left(2+\cos \left(\frac{k_{1} x}{L}\right)\right) \exp \left(-\frac{k_{2} \ln (2) x^{2}}{L^{2}}\right)
$$


where $k_{2}=100$ and $k_{1}=1.7 k_{2}$. The wave is convected via Eq.(18) over the range $-0.5 L \leq x \leq L$. The exact solution is obtained by

$$
f_{\text {exact }}(x, t)=f_{\infty}\left(2+\cos \left(\frac{k_{1} \hat{x}}{L}\right)\right) \exp \left(-\frac{k_{2} \ln (2) \hat{x}^{2}}{L^{2}}\right)
$$

where $\hat{x}=x-c_{\infty} t$. Since the wave pulse is initialised within the domain, nothing will pass through the inlet boundary. For this reason the interior schemes can be applied at the inlet boundary points $(i=\{0,1,2\})$ with the following boundary condition [1]:

$$
f(x<-0.5 L, t)=f^{\prime}(x<-0.5 L, t)=0 \text { for } t \geq 0
$$

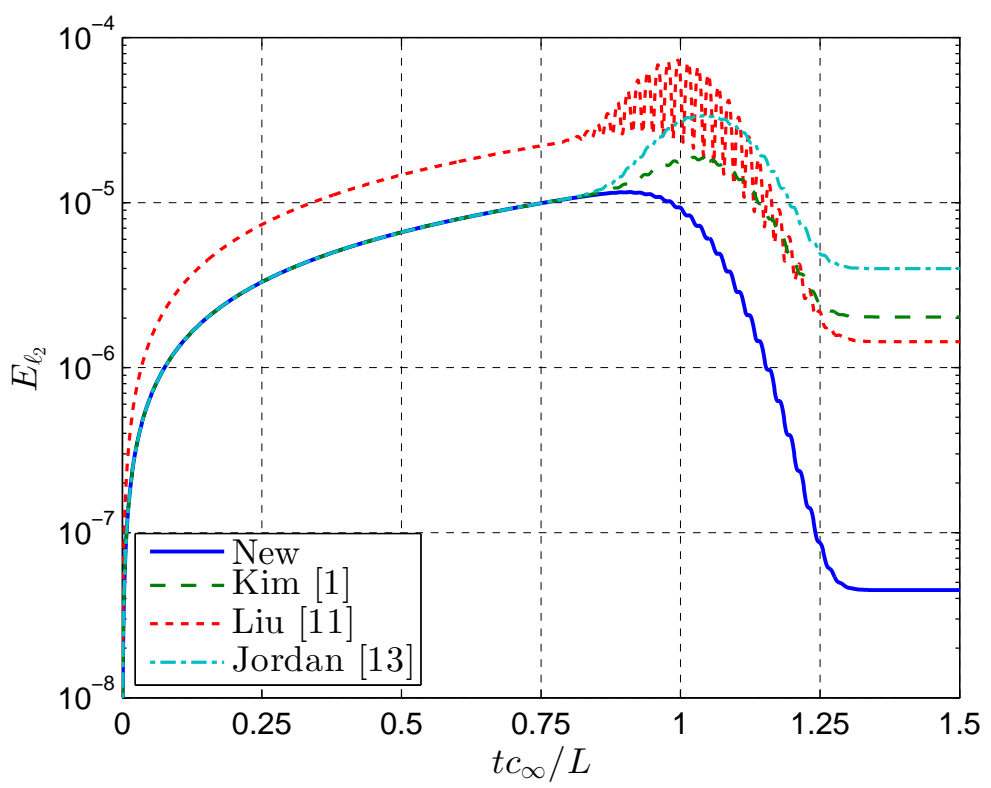

Figure 12: Time history of $\ell_{2}$-norm error produced by various schemes in calculation of the $1 \mathrm{D}$ scalar wave, $N=1000, \mathrm{CFL}=0.5$

The boundary schemes can then be applied and tested at the outlet 
boundary nodes $(i=\{N, N-1, N-2\})$ by measuring the error as the wave pulse leaves the domain at the non-dimensional time $t c_{\infty} / L=1.0$. Errors produced by the current schemes are compared to those produced by the schemes of Kim [1], Jordan [13] and Liu et al. [11]. Results are firstly presented without the assistance of compact filters, then comparisons are made to their filtered counterparts, thus demonstrating each templates sensitivity to the filtering process. The newly optimised schemes use the new boundary weighting factors suggested in section 4.2 with a filter cut-off of $\Omega_{c}=0.88 \pi$, while the other schemes maintain the original filter coefficients suggested in $[16]$.

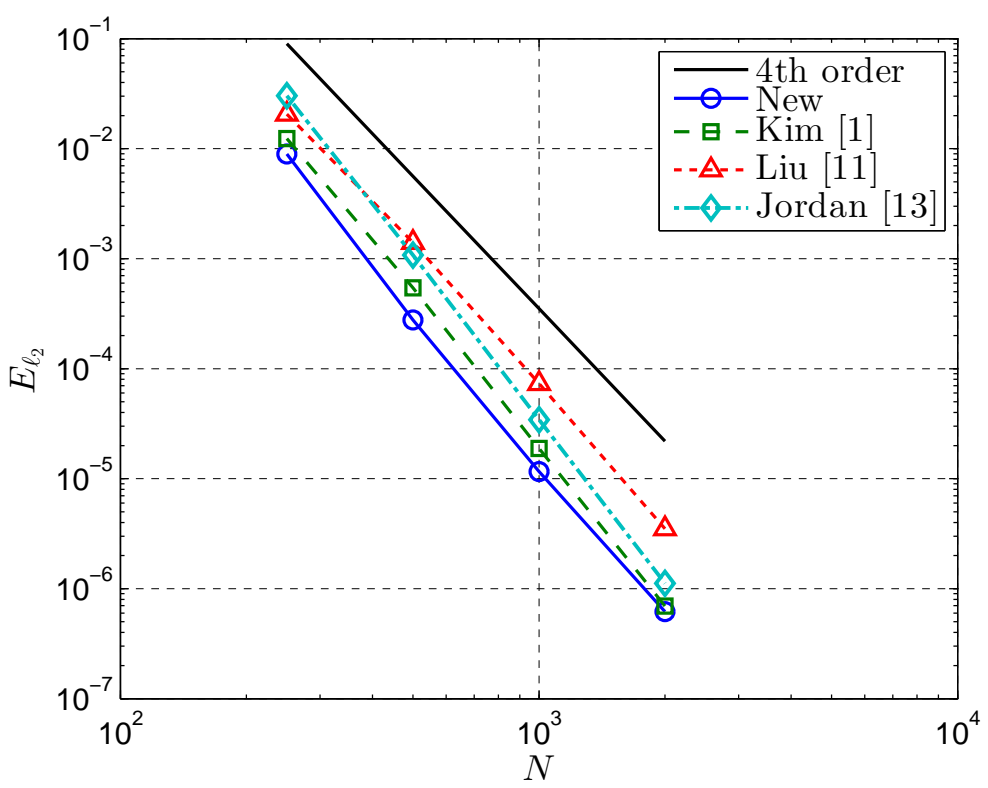

Figure 13: Maximum $\ell_{2}$-norm errors produced in the $1 \mathrm{D}$ scalar wave convection problem by various schemes at different grid levels, $\mathrm{CFL}=0.5$

Figure 11 shows a comparison of the wave produced by the current schemes and the analytical solution at three instances of time for a $\mathrm{CFL}=0.5$ 
and $N=1000$. The numerical result contains no perceivable errors, even as the wave leaves the domain exit boundary at $t c / L=1.0$. Figure 12 show the time histories of the $\ell_{2}$-norm error at a grid size of $N=1000$. The present schemes exclusively exhibit no overshoot as the wave leaves the domain. This corresponds to peak error reductions of $38.4 \%, 66.1 \%$ and $84.1 \%$ compared to that produced by the coefficients of Kim [1], Jordan [13] and Liu et al. [11], respectively. Another important quality, particularly for aeroacoustic simulations is that the final error tends to zero after the wave has left the domain. In this regard the result provided by the current schemes again outperforms that of previous studies resulting in a final error reduction of $97.8 \%$ compared to Kim [1], 98.9\% compared to Jordan [13] and 96.9\% compared to Liu et al. [11]. Figure 13 shows the maximum $\ell_{2}$-norm errors produced by each scheme at various grid levels. This confirms that the new schemes maintain the desired fourth-order convergence rate, while also achieving the lowest errors on all grid levels.

A Comparison between the $\ell_{2}$-norm error histories produced with and without compact filtering is shown in Figure 14 for $N=1000$. After filtering a comparable peak error level is achieved by the newly optimised schemes, Kim's schemes and Jordan's schemes. Liu's schemes on the other hand still manifests a significant overshoot at $t c_{\infty} / L=1.0$. The most robust performance is attained by the new schemes, which maintain similar error levels with and without filtering. In fact, they are the only schemes for which the peak error is slightly increased by filtering, suggesting that they are successful in resolving a broader range of scales. Conversely the schemes of Kim and Jordan prove to be highly susceptible to the filtering operations, therefore 

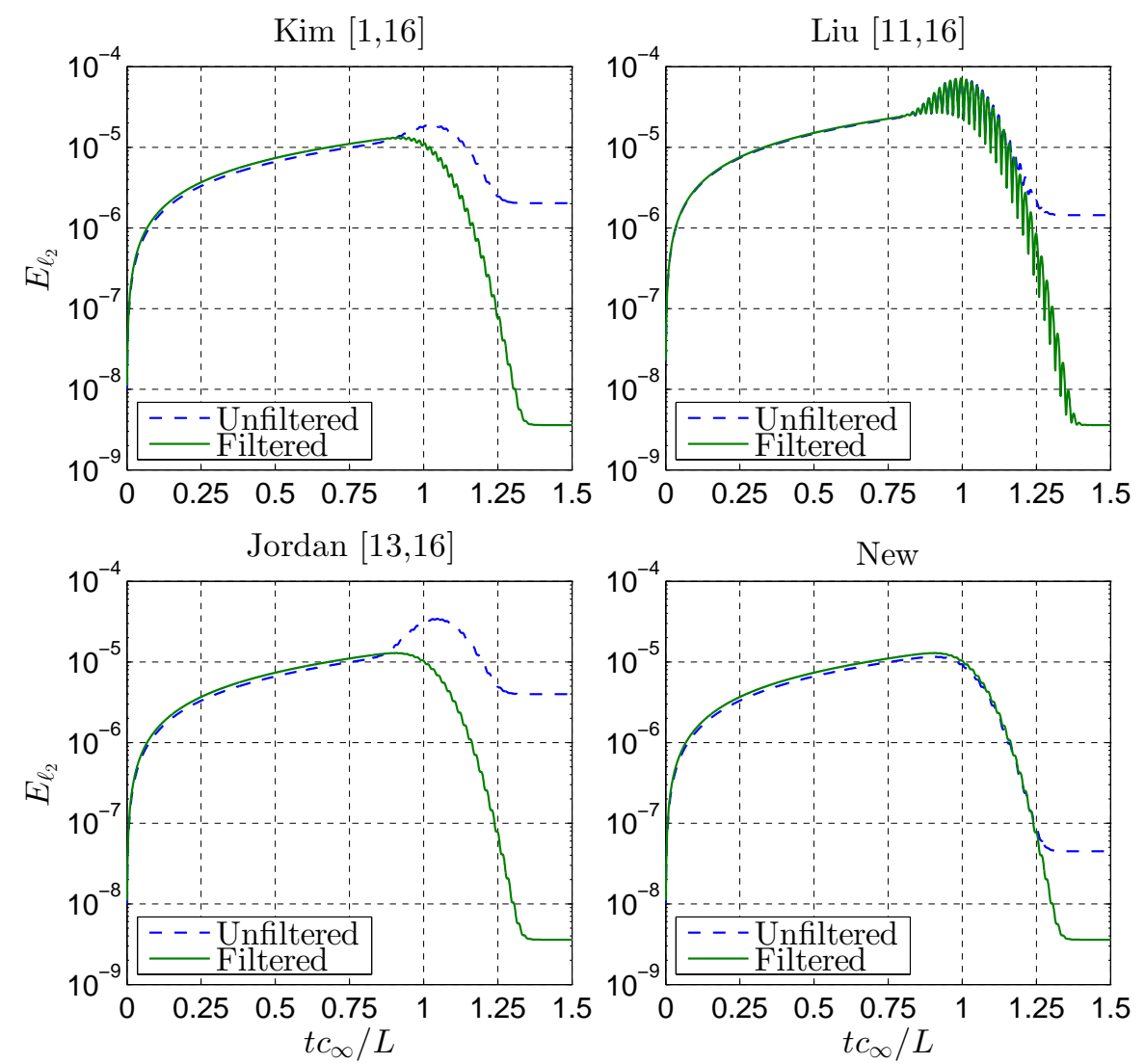

Figure 14: Comparison between $\ell_{2}$-norm error histories with and without filtering, $N=$ $1000, \mathrm{CFL}=0.5$. When filtering is implemented similar performance is obtained by the new schemes and those of Kim [1] and Jordan [13] 
extra caution should be exercised while selecting the filter cut-off wavenumber. The maximum $\ell_{2}$-norm errors produced by the new schemes with and without filtering is shown in Figure 15. Demonstrating that the similarity between filtered and unfiltered solutions is consistent over a range of grid levels.

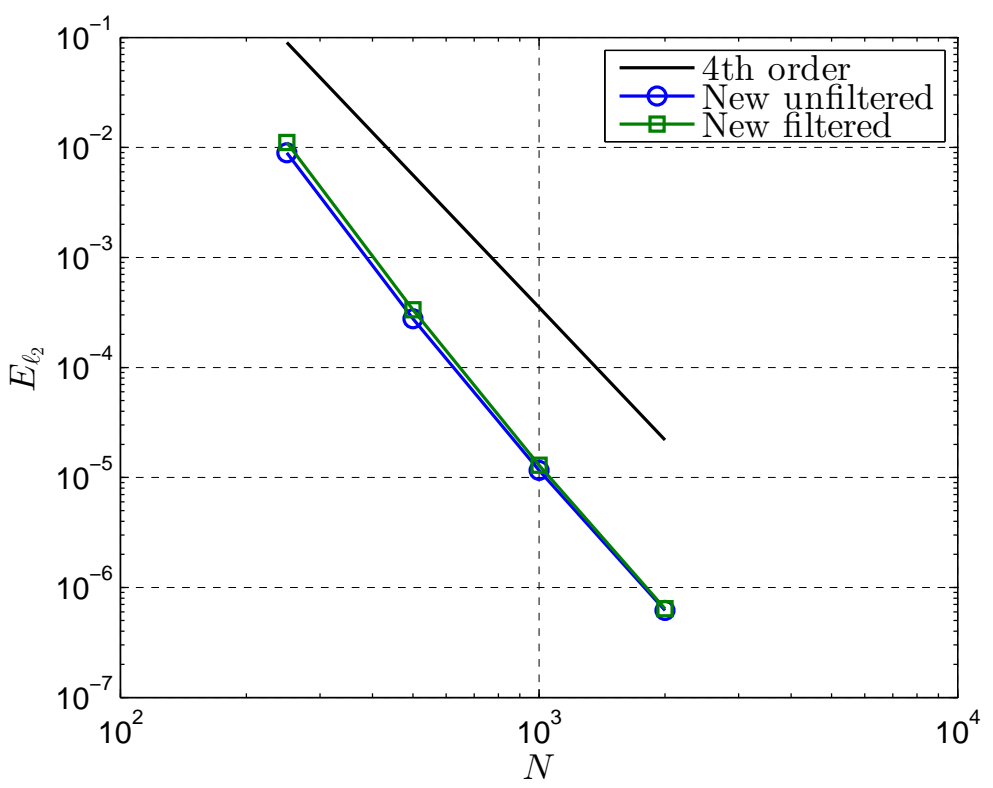

Figure 15: Comparison of maximum $\ell_{2}$-norm errors produced in the $1 \mathrm{D}$ scalar wave convection problem by the current scheme, with and without filtering, $\mathrm{CFL}=0.5$

\subsection{Two-dimensional Inviscid Vortex Convection}

In this problem the 2D compressible Euler equations are solved in full conservative form, in order to simulate the convection of an inviscid 2D vorticity wave in a supersonic flow. This problem was originally proposed by Yee et al. [19] to validate their high-order shock capturing scheme and filters. 
The governing equations are described as follows

$$
\frac{\partial \mathbf{Q}}{\partial t}+\frac{\partial \mathbf{E}}{\partial x}+\frac{\partial \mathbf{F}}{\partial y}=\mathbf{0}
$$

where $\mathbf{Q}, \mathbf{E}$ and $\mathbf{F}$ represent the following:

$$
\mathbf{Q}=\left(\begin{array}{c}
\rho \\
\rho u \\
\rho v \\
\rho e_{t}
\end{array}\right), \quad \mathbf{E}=\left(\begin{array}{c}
\rho u \\
\rho u^{2}+p \\
\rho u v \\
\rho\left(e_{t}+p\right) u
\end{array}\right) \text { and } \quad \mathbf{F}=\left(\begin{array}{c}
\rho v \\
\rho u v \\
\rho v^{2}+p \\
\rho\left(e_{t}+p\right) v
\end{array}\right) \text {. }
$$

where $\rho, u, v$ and $p$ are the primitive variables (density, streamwise velocity, vertical velocity and pressure), and subscript $\infty$ represents free-stream conditions. The total energy per unit mass is given by $e_{t}=p /[(\gamma-1) \rho]+\left(u^{2}+v^{2}\right) / 2$, and $\gamma=c_{p} / c_{v}$ is the ratio of specific heats, set to $\gamma=1.4$ for air. The calculation is carried out with the following initial conditions

$$
\begin{aligned}
& \left.\begin{array}{l}
\frac{\rho(x, y)}{\rho_{\infty}}=\left(1-\frac{\gamma-1}{2} \psi^{2}(x, y)\right)^{\frac{1}{\gamma-1}} \\
\frac{u(x, y)}{a_{\infty}}=M_{\infty}+K y \psi(x, y) \\
\frac{v(x, y)}{a_{\infty}}=-K x \psi(x, y)
\end{array}\right\}, \text { for }\left\{\begin{array}{l}
-0.5 L \leq x \leq 2.5 L \\
-0.75 L \leq y \leq 0.75 L
\end{array}\right. \\
& \frac{p(x, y)}{p_{\infty}}=\left(\frac{\rho}{\rho_{\infty}}\right)^{\gamma}
\end{aligned}
$$


with

$$
\psi(x, y)=\frac{\epsilon}{2 \pi} \exp \left(\frac{1}{2}\left(1-K^{2}\left(x^{2}+y^{2}\right)\right)\right),
$$

where $K=1 / R$ and $R=0.08 L$, which represents the radius of the vortex. The vortex strength is controlled by the parameter $\epsilon . \epsilon=0.1$ corresponds to a linear case, while higher values correspond to more non-linear cases. The free stream velocity is defined as $u_{\infty}=M_{\infty} a_{\infty}$, with the Mach number $M_{\infty}=2$, and the ambient speed of sound $a_{\infty}=\sqrt{\gamma p_{\infty} / \rho_{\infty}}$. As there is a supersonic free stream velocity, downstream disturbances will have no influence on upstream flow properties. Therefore boundary conditions need not be applied at the domain outlet. Furthermore this advocates the use of interior schemes at the first three inlet boundary points, since the $x$ derivatives in Eq.(32) may be set to zero prior the inlet boundary. In addition to the domain outlet, boundary schemes are applied to the top $(j=\{N-$ $2, N-1, N\})$ and bottom edges $(j=\{0,1,2\})$ of the grid with the nonreflective boundary conditions suggested in [20]. The compact filter cut-off frequency of $\Omega_{c}=0.88 \pi$ is used with the boundary weighting factors to ensure a numerical stable solution is obtained. As before time integration is carried out with classical fourth-order Runge-Kutta method, until a non-dimensional time of $u_{\infty} t / L=1.5$ using $C F L=0.5$.

In order to identify the errors generated at the exit boundary the solution generated on the domain $-0.5 L \leq x \leq 2.5 L,-0.75 L \leq y \leq 0.75 L$ is treated as a reference solution. This is compared to the result obtained on a grid truncated by a factor of 2 in the streamwise direction $(-0.5 L \leq x \leq L$, $-0.75 L \leq y \leq 0.75 L)$. At $u_{\infty} t / L=1.0$ the core of the propagating vortex will have reached the truncated domain exit boundary, but will still be well 
within the interior region of the full length domain. By comparing solutions at this instant of time the accuracy of the boundary schemes can be determined. Further justification for this approach is provided in Appendix A. A two-dimensional equivalent of the $\ell_{2}$-norm error, based on corresponding grid points of the truncated and full length domains can be defined as follows

$$
E_{\ell_{2}}\left(t_{s}\right)=\left(\frac{L^{2}}{\left[(N+1) \epsilon u_{\infty}\right]^{2}} \sum_{i=0}^{N} \sum_{j=0}^{N}\left(\nu_{i, j}^{F}-\nu_{i, j}^{T}\right)^{2}\right)^{1 / 2}
$$
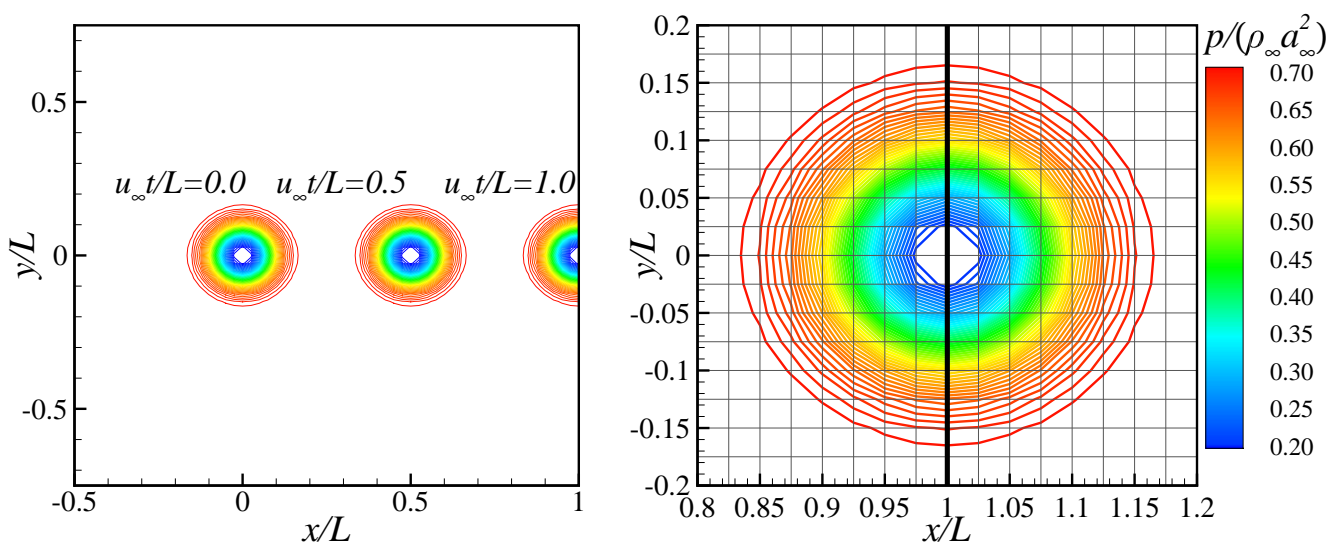

Figure 16: Left: Contours of normalised pressure for the vortex convection problem obtained by the new schemes at three instances of time with $\epsilon=5$. The domain is truncated by a factor of 2 in the streamwise direction such that a computational exit boundary exists at $x=1.0 \mathrm{~L}$, where boundary schemes are applied. A total of $60 \times 60$ grid cells are used for the computation with a uniform grid spacing of $\Delta x=0.025 L$. Right: Comparison of normalised pressure contours around $x=1.0 L$ for the truncated domain and a full length domain where the exit boundary is further downstream. The full length domain maintains the same grid spacing and hence consists of $120 \times 60$ grid cells

where $\nu$ is a normalised primitive variable $\left(u / a_{\infty}, v / a_{\infty}, \rho / \rho_{\infty}, p /\left(\rho_{\infty} a_{\infty}^{2}\right)\right)$, $(N+1)^{2}$ is the number of grid points contained within the truncated grid, $t_{s}$ is the current time step and superscripts $F$ and $T$ correspond to the full 
length and truncated domains. All numerical errors are compared to those produced using the coefficients suggested by $\operatorname{Kim}[1,16]$.

Figure 16 show contours of normalised pressure obtained at three instances of time $u_{\infty} t / L=0,0.5,1.0$ on a truncated $60 \times 60$ grid. There are no observable deformations as the vortex leaves the exit boundary at $x=1.0 \mathrm{~L}$. Also shown is a comparison of the solution produced on both the truncated $(60 \times 60$ grid $)$ and reference grids $(120 \times 60$ grid $)$ at $u_{\infty} t / L=1.0$. Despite the fact that this result is obtained on a very coarse grid with the most non-linear vortex strength $(\epsilon=5)$, the two results remain consistent.

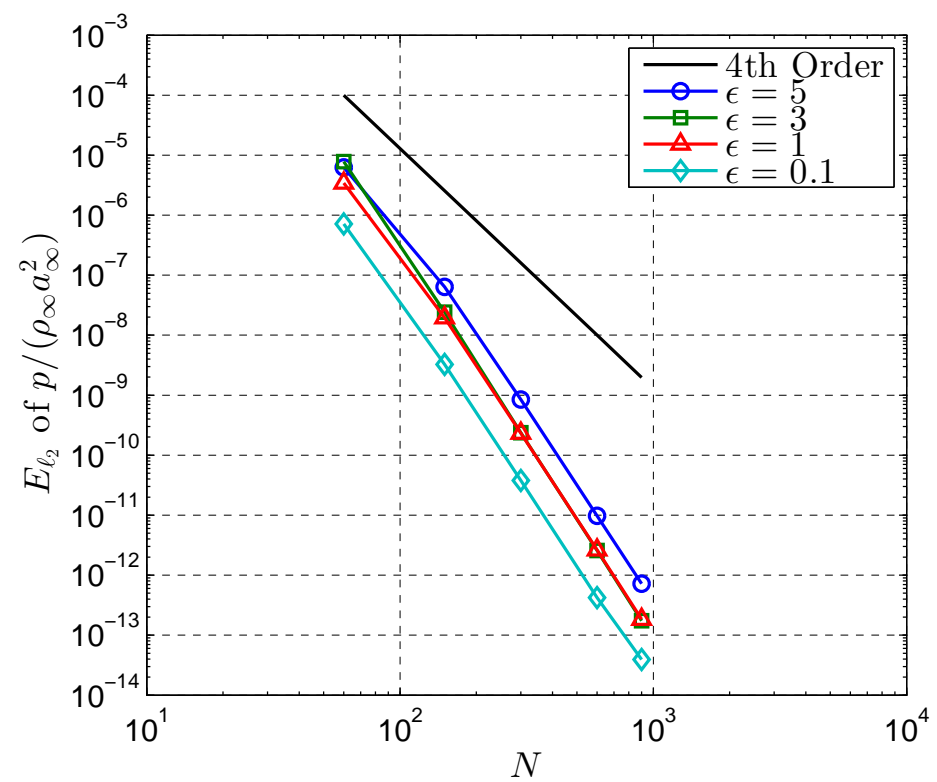

Figure 17: $\ell_{2}$-norm errors based on $p /\left(\rho_{\infty} a_{\infty}^{2}\right)$ produced at $u_{\infty} t / L=1.0$ by the new schemes during the vortex convection problem. Results shown with $\epsilon=0.1,1,3$ and 5 at various grid levels

Figure 17 shows the convergence rates of the normalised pressure $\ell_{2^{-}}$ norm error produced at $u_{\infty} t / L=1.0$ with various values of $\epsilon$. For each grid 
level the grid spacing is kept uniform in both the streamwise and vertical directions. The current schemes successfully exceed the desired fourth-order convergence rate for both linear and non-linear vortex cases. Comparisons to the previous study $[1,16]$ are also given in Figure 18 for each primitive variable and $\epsilon=5$. Large error reductions are achieved by the new schemes
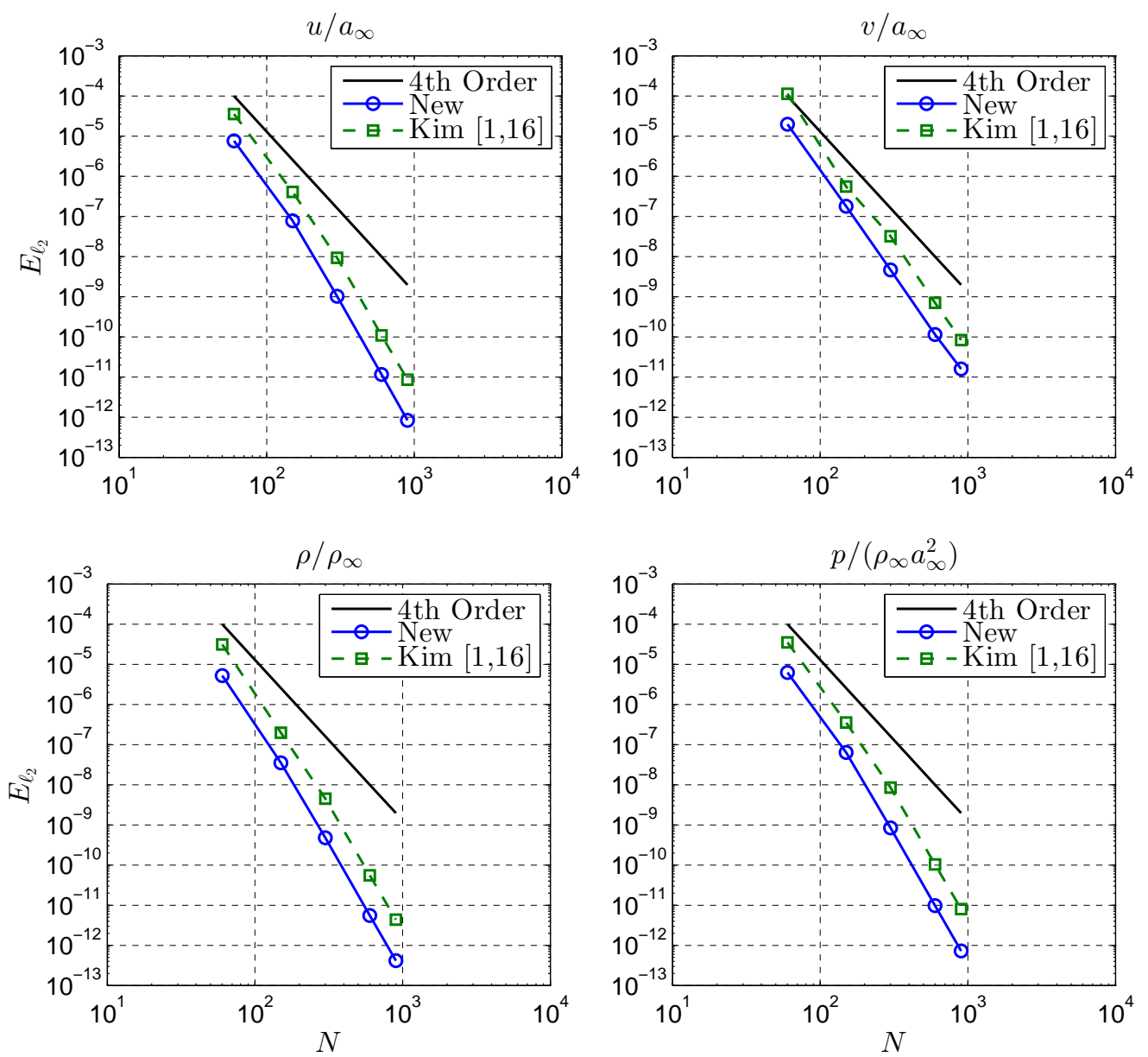

Figure 18: Primitive variable $\ell_{2}$-norm errors generated by the new schemes and those of Kim $[1,16]$ during the vortex convection problem with an increasing number of nodes. Errors calculated at $u_{\infty} t / L=1.0$ with $\epsilon=5$

for all primitive variables and grid levels, in some cases in excess of to an order 
of magnitude. On average errors are reduced by a factors of 8.31,7.72, 5.42 and 8.58 for $u / a_{\infty}, v / a_{\infty}, \rho / \rho_{\infty}$ and $p /\left(\rho_{\infty} a_{\infty}^{2}\right)$ respectively across the five grid levels tested. This clearly demonstrates that the present schemes are also capable of large accuracy improvements in multidimensional problems.

Further comparisons for the error based on normalised pressure are shown in Figure 19 for $u_{\infty} t / L=1.0$ obtained with different vortex strengths. Using a $60 \times 60$ grid error reductions range from $60.1 \%-82.2 \%$, while for a $300 \times 300$ grid they fall between $88.6 \%-91.3 \%$. In Figure 20 comparisons are also made with the schemes of Liu et al. [11] and Jordan [13] for the error time history using a $60 \times 60$ grid and $\epsilon=5$. For each case compact filtering is employed to ensure stable solutions. The results are shown firstly with a filter cut-off of $\Omega_{c}=0.88 \pi$ utilising the boundary weighting strategy in Eq.(17). The new schemes are successful in obtaining the lowest errors during the simulation. A similar performance is also achieved for the schemes of Jordan [13], however as demonstrated in the previous one-dimensional benchmark problem the low errors produced by Jordan's template were not maintained when the filter cut-off was increased. With a higher filter cut-off (globally set to $\left.\Omega_{c}=0.95 \pi\right)$ the error produced by Jordan's schemes increases, whereas for the new schemes it is reduced, thus resulting in a more substantial error reduction offered by the new schemes.

\subsection{Deformed Grid Two-dimensional Inviscid Vortex Convection}

In this benchmark problem the performance of the current schemes on curvilinear grids is analysed by revisiting the two-dimensional inviscid vortex convection problem. The original uniform grid is deformed by implementing the following equations [21] 

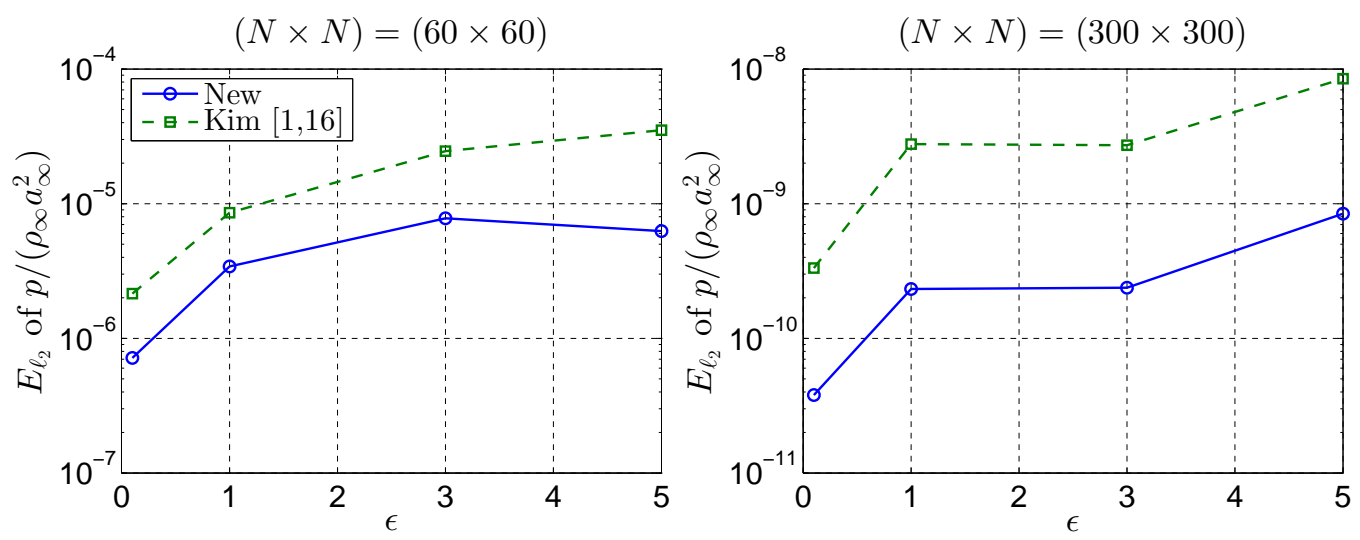

Figure 19: Comparison of $\ell_{2}$-norm errors based on $p /\left(\rho_{\infty} a_{\infty}^{2}\right)$ produced during the vortex convection problem. Obtained at $u_{\infty} t / L=1.0$, with various vortex strengths $(\epsilon)$. Left: $60 \times 60$ grid. Right: $300 \times 300$ grid
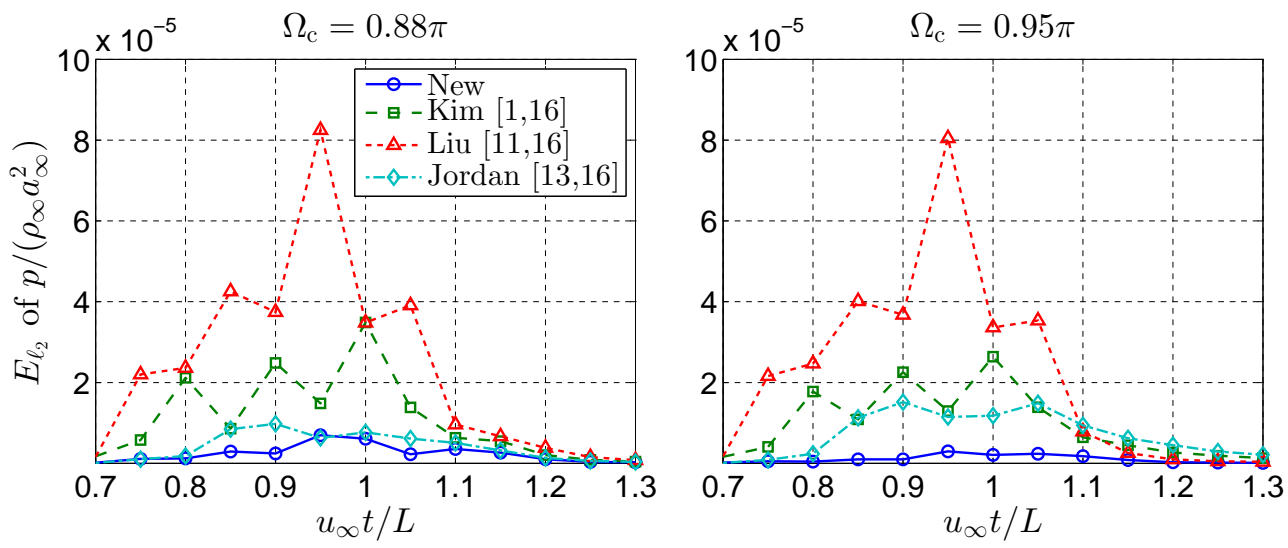

Figure 20: Time history of $\ell_{2}$-norm errors based on $p /\left(\rho_{\infty} a_{\infty}^{2}\right)$ produced during the vortex convection problem with $N \times N=60 \times 60$ and $\epsilon=5$. Left: filter cut-off $\Omega_{c}=0.88 \pi$ utilising the boundary weighting strategy in Eq.(17) $\left(w_{2}=0.085\right.$ previous schemes, $w_{2}=0.0485$ new schemes). Right: global filter cut-off $\Omega_{c}=0.95 \pi, w_{2}=0$ 


$$
\begin{aligned}
& x_{i, j}=-\frac{L}{2}+\frac{3 L}{2}\left[\frac{i}{N}+\mu \sin \left(\frac{4 \pi j}{N}\right)\right] \\
& y_{i, j}=-\frac{3 L}{4}+\frac{3 L}{2}\left[\frac{j}{N}+\mu \sin \left(\frac{4 \pi i}{N}\right)\right]
\end{aligned}
$$

where $\mu$ determines the amount of grid deformation. $\mu=0$ would revert the grid to the uniform case analysed in the previous section. As before the problem consists of solving the compressible two-dimensional Euler equations, although this time in a generalised coordinate system

$$
\frac{\partial \widehat{\mathbf{Q}}}{\partial t}+\frac{\partial \widehat{\mathbf{E}}}{\partial \xi}+\frac{\partial \widehat{\mathbf{F}}}{\partial \eta}=\mathbf{0},
$$

with

$$
\widehat{\mathbf{Q}}=\mathbf{Q} / J, \quad \widehat{\mathbf{E}}=\left(\xi_{x} \mathbf{E}+\xi_{y} \mathbf{F}\right) / J, \quad \widehat{\mathbf{F}}=\left(\eta_{x} \mathbf{E}+\eta_{y} \mathbf{F}\right) / J
$$

where $\xi_{x, y}$ and $\eta_{x, y}$ are the grid metrics, and $J^{-1}=\left(x_{\xi} y_{\eta}=x_{\eta} y_{\xi}\right)$ is the Jacobian determinant of the transformation. Since the finite-difference template is also required to calculate the grid metric this represents a more thorough test of their performance. The calculations are run using the most non-linear vortex case $(\epsilon=5), C F L=0.5$, and $M_{\infty}=2.0$. The compact filtes are also implemented utilising $\Omega_{c}=0.88 \pi$ and the appropiate boundary weighting factors. The $\ell_{2}$ norm errors are once again evaluated based on Eq.(36) as the vortex leaves the exit boundary at $x=1.0 L$. Figure 21 shows contours on normalised spanwise vorticity $\left(\omega_{z} L /\left(a_{\infty} \epsilon\right)\right.$ where $\left.\omega_{z}=\partial v / \partial x-\partial u / \partial y\right)$ at three instances of time $\left(u_{\infty} t / L=0,0.5\right.$ and 1.0). The truncated domain grid consists of $N \times N=100 \times 100$ grid cells and utilises $\mu=0.05$ (1 in 2 grid 
points shown). At $u_{\infty} t / L=1.0$ the vortex is halfway through the truncated domain exit boundary. At this point there is no noticable deformation to the vortex or disimilarity with the full domain solution.

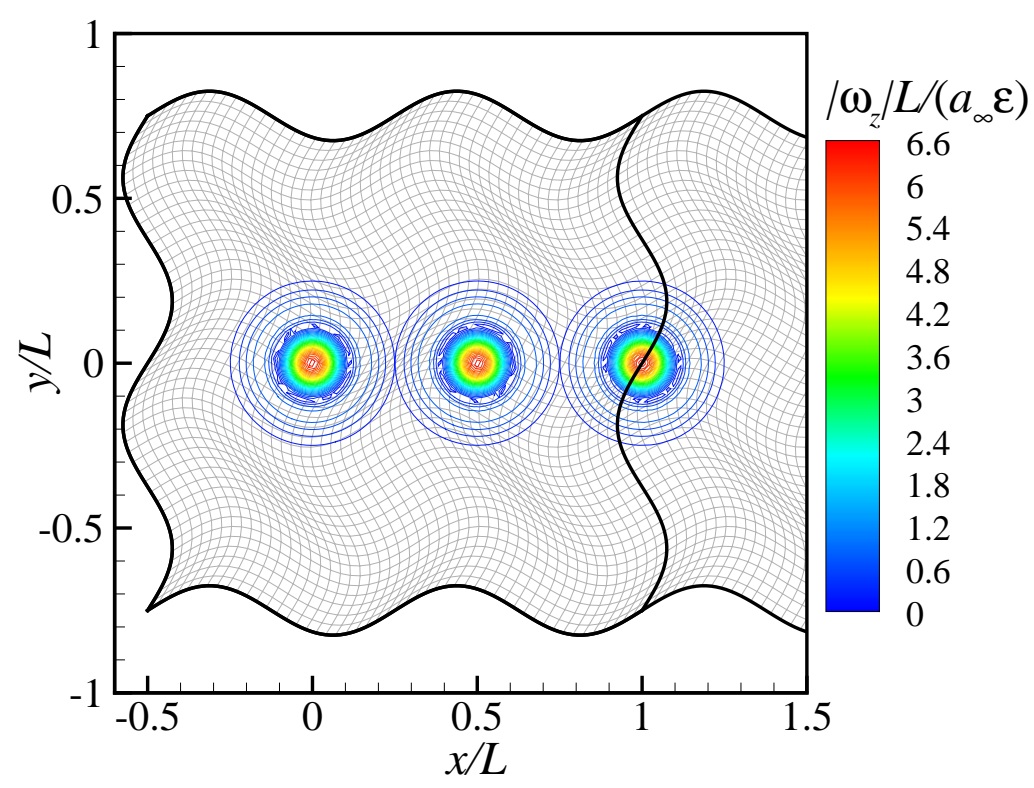

Figure 21: Contours of normalised spanwise vorticity magnitude for the vortex convection problem utilising a curvilinear (deformed) grid. The grid (generated by Eq.(37)) consists of $100 \times 100$ grid cells with a uniform spacing and $\mu=0.05$ ( 1 in 2 grid lines shown)

The maximum $\ell_{2}$ norm error convergence is shown in Figure 22 for the new schemes and those of $\operatorname{Kim}[1,16]$ based on normalised pressure $\left(p /\left(\rho_{\infty} a_{\infty}^{2}\right)\right.$. This demonstrates that the new schemes are able to maintain the desired 4th-order convergence rate on heavily deformed curivlinear grids. The error reduction produced by the new schemes increases with $N$ ranging from $52.16 \%$ for the coarsest grid, to $94.63 \%$ for the finest. Figure 23 shows the $\ell_{2}$ norm error time history produced by the new schemes and those of Kim [1], Liu Liu et al. [11] and Jordan Jordan [13]. Each scheme is used in conjuction 
with the compact filtering [16], with results shown for the $100 \times 100$ grid. The new schemes achieve a 12.6, 7.2 and 3.0 times improvement to the maximum error produced during the calculation compared to the schemes of Liu et al. [11], Kim [1] and Jordan [13] respectively.

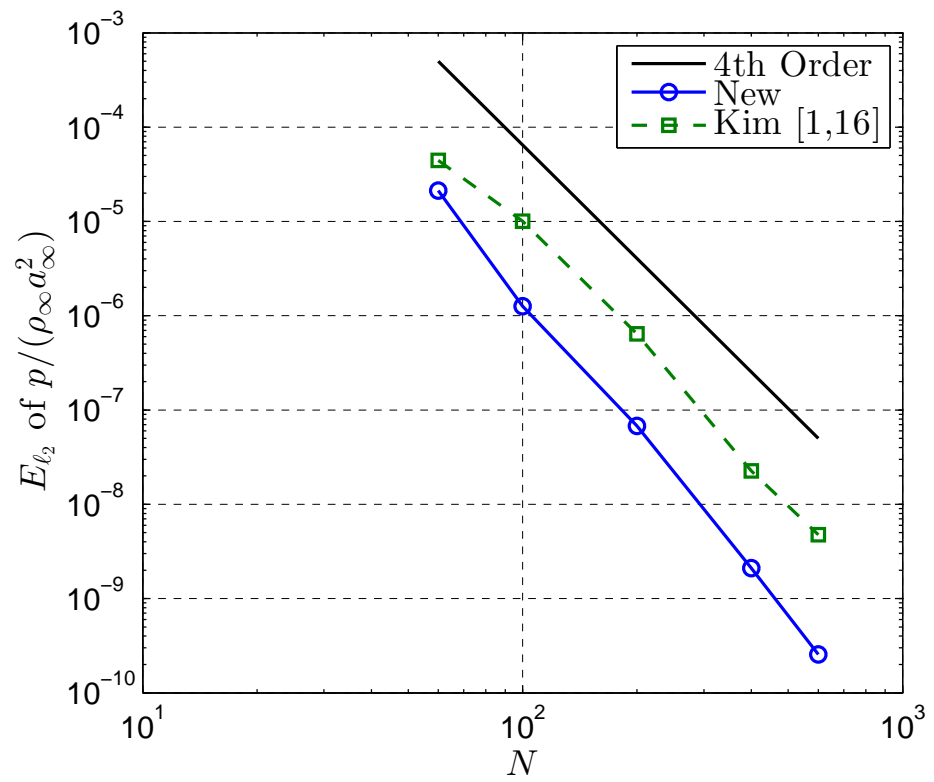

Figure 22: $\ell_{2}$-norm errors based on $p /\left(\rho_{\infty} a_{\infty}^{2}\right)$ produced at $u_{\infty} t / L=1.0$ by the new schemes and those of Kim [1, 16] during the deformed grid vortex convection problem with $\mu=0.05$. Results shown with $\epsilon=5$ at various grid levels

\section{Pseudo-boundary Schemes}

Thus far we have concentrated on reducing the total resolution error between the composite template and exact differentiation. Another potential target for improvement is the relative error between consecutive points in the composite template. This is a particular concern between the final central interior node and the first non-central boundary node, where typically a 
sharp degradation in the spectral properties are observed. The approach taken in this section is to retune the coefficients of the first few interior nodes, such that they ease this performance discontinuity, and thus achieve a higher accuracy. The retuned interior schemes are herein referred to as pseudo-boundary schemes. The coefficient matrices $\mathbf{P}$ and $\mathbf{Q}$ are updated to include the pseudo-boundary schemes at nodes $i=\{3, N-2\}, i=\{4, N-1\}$ and $i=\{5, N\}$ are displayed below. Hatted variables denote the pseudoboundary coefficients.

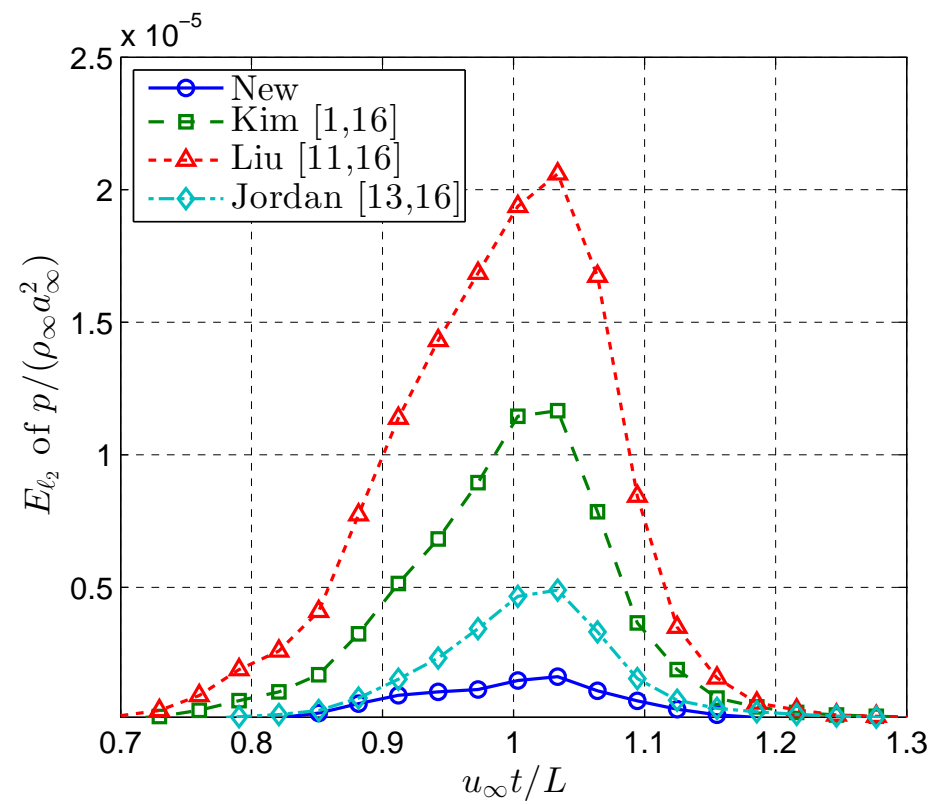

Figure 23: Time history of $\ell_{2}$-norm errors based on $p /\left(\rho_{\infty} a_{\infty}^{2}\right)$ produced by various schemes during the deformed grid vortex convection problem using $\epsilon=5$. The grid consists of $N \times N=100 \times 100$ grid cells with $\mu=0.05 . \Omega_{c}=0.88 \pi$ 


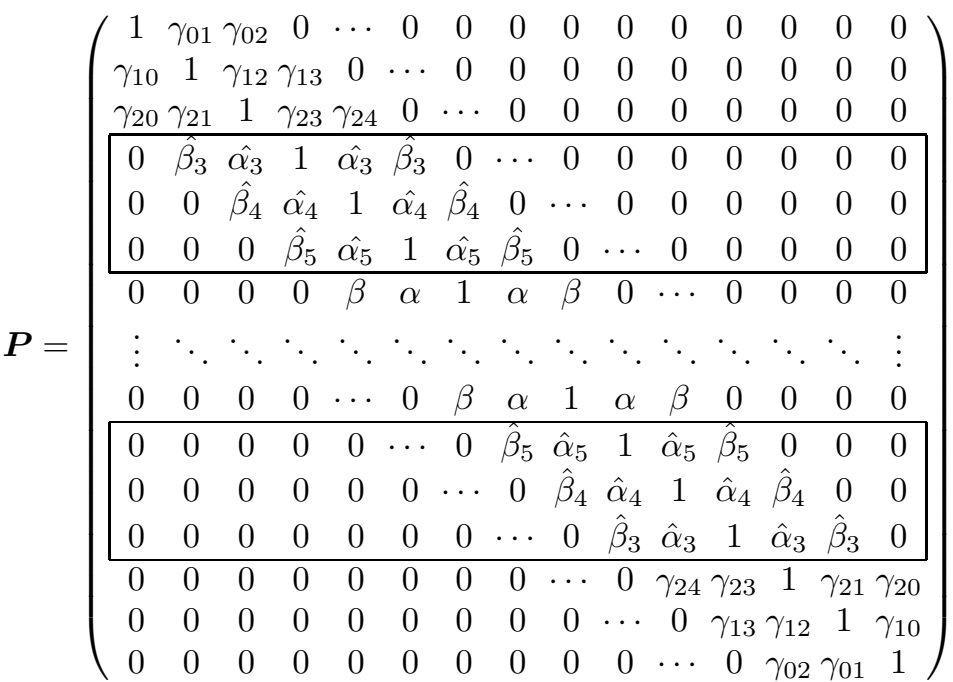

$\boldsymbol{Q}=\left(\begin{array}{ccccccccccccccc}b_{00} & b_{01} & b_{02} & b_{03} & b_{04} & b_{05} & b_{06} & 0 & 0 & 0 & 0 & 0 & 0 & \cdots & 0 \\ b_{10} & b_{11} & b_{12} & b_{13} & b_{14} & b_{15} & b_{16} & 0 & 0 & 0 & 0 & 0 & 0 & \cdots & 0 \\ b_{20} & b_{21} & b_{22} & b_{23} & b_{24} & b_{25} & b_{26} & 0 & 0 & 0 & 0 & 0 & 0 & \cdots & 0 \\ -\hat{a}_{33}-\hat{a}_{23} & -\hat{a}_{13} & 0 & \hat{a}_{13} & \hat{a}_{23} & \hat{a}_{33} & 0 & 0 & 0 & 0 & 0 & 0 & \cdots & 0 \\ 0 & -\hat{a}_{34} & -\hat{a}_{24} & -\hat{a}_{14} & 0 & \hat{a}_{14} & \hat{a}_{24} & \hat{a}_{34} & 0 & 0 & 0 & 0 & 0 & \cdots & 0 \\ 0 & 0 & -\hat{a}_{35}-\hat{a}_{25} & -\hat{a}_{15} & 0 & \hat{a}_{15} & \hat{a}_{25} & \hat{a}_{35} & 0 & 0 & 0 & 0 & \cdots & 0 \\ \hline 0 & 0 & 0 & -a_{3} & -a_{2} & -a_{1} & 0 & a_{1} & a_{2} & a_{3} & 0 & 0 & 0 & \cdots & 0 \\ \vdots & \ddots & \ddots & \ddots & \ddots & \ddots & \ddots & \ddots & \ddots & \ddots & \ddots & \ddots & \ddots & \ddots & \vdots \\ 0 & \cdots & 0 & 0 & 0 & -a_{3} & -a_{2} & -a_{1} & 0 & a_{1} & a_{2} & a_{3} & 0 & 0 & 0 \\ \hline 0 & \cdots & 0 & 0 & 0 & 0 & -\hat{a}_{35}-\hat{a}_{25}-\hat{a}_{15} & 0 & \hat{a}_{15} & \hat{a}_{25} & \hat{a}_{35} & 0 & 0 \\ 0 & \cdots & 0 & 0 & 0 & 0 & 0 & -\hat{a}_{34}-\hat{a}_{24}-\hat{a}_{14} & 0 & \hat{a}_{14} & \hat{a}_{24} & \hat{a}_{34} & 0 \\ 0 & \cdots & 0 & 0 & 0 & 0 & 0 & 0 & -\hat{a}_{33}-\hat{a}_{23}-\hat{a}_{13} & 0 & \hat{a}_{13} & \hat{a}_{23} & \hat{a}_{33} \\ \hline 0 & \cdots & 0 & 0 & 0 & 0 & 0 & 0 & -b_{26} & -b_{25} & -b_{24}-b_{23}-b_{22}-b_{21}-b_{20} \\ 0 & \cdots & 0 & 0 & 0 & 0 & 0 & 0 & -b_{16} & -b_{15} & -b_{14}-b_{13}-b_{12}-b_{11}-b_{10} \\ 0 & \cdots & 0 & 0 & 0 & 0 & 0 & 0 & -b_{06} & -b_{05} & -b_{04}-b_{03}-b_{02}-b_{01}-b_{00}\end{array}\right)$

Since maximising the spectral resolution properties of pseudo-boundary schemes is not necessarily the objective, optimisation via the wavenumber error fitness function is avoided. Alternatively, the pseudo-boundary coefficients are tuned through direct application to the second benchmark problem (two-dimensional inviscid vortex convection). Five equations are required 
to determine the five compact finite difference coefficients of each pseudoboundary scheme. The first two are formed by the requirement for fourth order truncation error, while the remaining three are determined by minimising an integral error function defined between exact and modified wavenumber curves. This process was utilised by Kim [1] to obtain the main interior scheme used throughout this paper. In this case the error function integration range $r$ is selected as the tuning parameter, and is varied between $0.7 \pi \leq r \leq \pi$. The coefficients of the $i=\{3, N-3\}$ nodes are the first to be modified. Firstly by replacing them with coefficients generated using $r=0.7 \pi$, and then by incrementing $r$ in steps of $\Delta r=0.01 \pi$. The $\ell_{2}$-norm error in the second benchmark problem based on normalised pressure, $\epsilon=5$ and a $N \times N=150 \times 150$ grid is then determined, and the most successful coefficients retained. The same process is then undertaken at the next node $(i=\{4, N-4\})$, this time initialising the search with the best coefficients obtained by the previous step. As well as reducing computational cost, this ensures that subsequent pseudo-boundary points will be based on a higher integration range, encouraging a gradual increase in spectral resolution. This process was continued until a minimum $\ell_{2}$-norm error was reached, resulting in pseudo-boundary coefficients applied to the $i=\{3, N-2\}, i=\{4, N-1\}$ and $i=\{5, N\}$ interior nodes, shown in Table 4 .

\subsection{Pseudo-boundary Modified Wavenumber Characteristics}

Figure 24 shows the respective dispersion and dissipation errors (Eqs.(23) and (24)) produced by a $N=13$ matrix system. This consists of 6 boundary, 6 pseudo-boundary and 1 main interior point. The dissipation error distributions behave generally as anticipated, gradually improving as $i$ is increased. 


\begin{tabular}{cccc}
\hline & $i=3$ & $i=4$ & $i=5$ \\
\hline$r$ & $0.710 \pi$ & $0.757 \pi$ & $0.920 \pi$ \\
$\hat{\alpha}_{i}$ & 0.56075605925645422 & 0.56692843691602146 & 0.60253603159337543 \\
$\hat{\beta}_{i}$ & 0.07930134421661057 & 0.08298645604575880 & 0.10724531301136056 \\
$\hat{a}_{1 i}$ & 0.66544805450470135 & 0.66024976364668564 & 0.62798918770826639 \\
$\hat{a}_{2 i}$ & 0.23030478868512835 & 0.23706131441853673 & 0.27695667488600395 \\
$\hat{a}_{3 i}$ & 0.00466659053270228 & 0.00518083349267372 & 0.00929293570815389 \\
\hline
\end{tabular}

Table 4: Pseudo-boundary scheme coefficients

Please note that the dissipation error produced at the interior node $(i=6)$ is zero, and therefore it is not shown here. (This is always the case for the central row of a composite template). The dispersion errors on the other hand are perhaps more surprising, particularly for the pseudo-boundary points. Over some wavenumber regions they manage to obtain even lower dispersion errors than the interior node. Despite this, the overall resolution error produced at these points will be larger due to their non-zero dissipation errors.
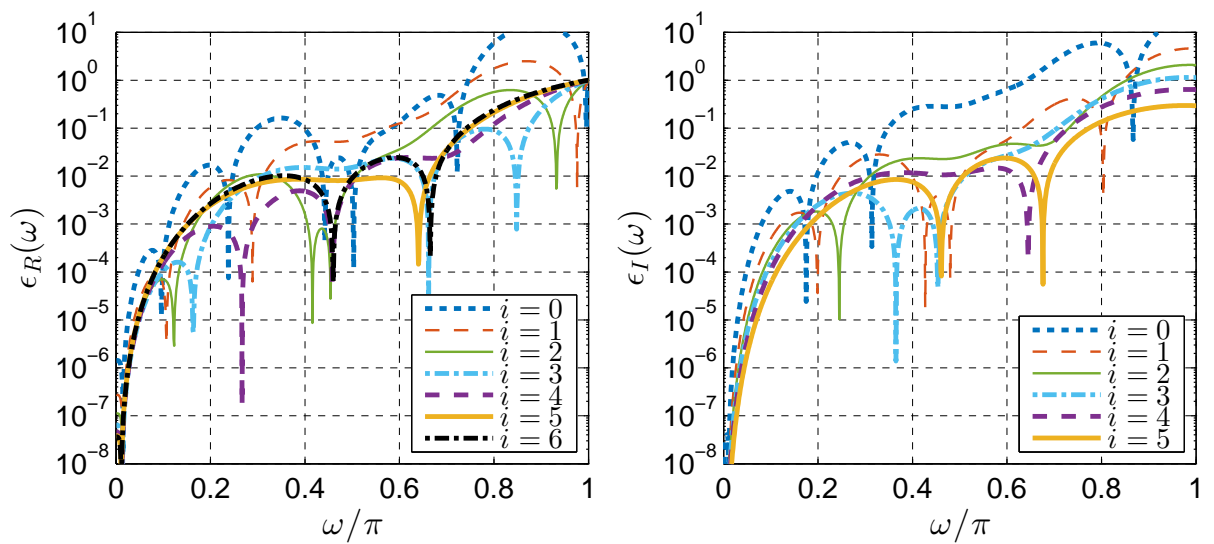

Figure 24: Wavenumber error plots for the current finite difference template and pseudoboundary schemes $(N=13)$ 


\subsection{Pseudo-boundary Performance}

An example of the accuracy improvements offered by pseudo-boundary schemes is given in Figure 25. This shows a comparison of the normalised pressure $\ell_{2}$-norm error history, obtained during the two-dimensional vortex convection problem using the coarsest grid and most non-linear vortex strength. The result is a significantly large reduction of $44.9 \%$ to the error at $u_{\infty} t / L=1.0$. Although it appears pseudo-boundary schemes have the potential to offer reasonably large accuracy improvements, they do present a challenge in terms of numerical stability. In the current benchmark test problems they were able to obtain stable solutions. However, they fail to meet eigenvalue requirements for linear stability unless an excessive level of filtering is applied. For this reason their stability in other problems cannot be ensured.

\section{Conclusion}

A new optimisation strategy for compact finite difference boundary schemes is successfully implemented utilising a genetic algorithm. This comprises of an objective function based upon a new formulation for the modified wavenumber of composite templates. A non-linear constraint for eigenvalue stability is used to ensure a stable matrix system is automatically obtained through compact filtering. Pentadiagonal schemes with a seven-point stencil are the primary focus, however a similar approach could be utilised for alternate compact schemes if desired. The optimised schemes provide substantial improvements to resolution, accuracy and computational efficiency in a series of one and two-dimensional benchmark problems. They are suitable for 


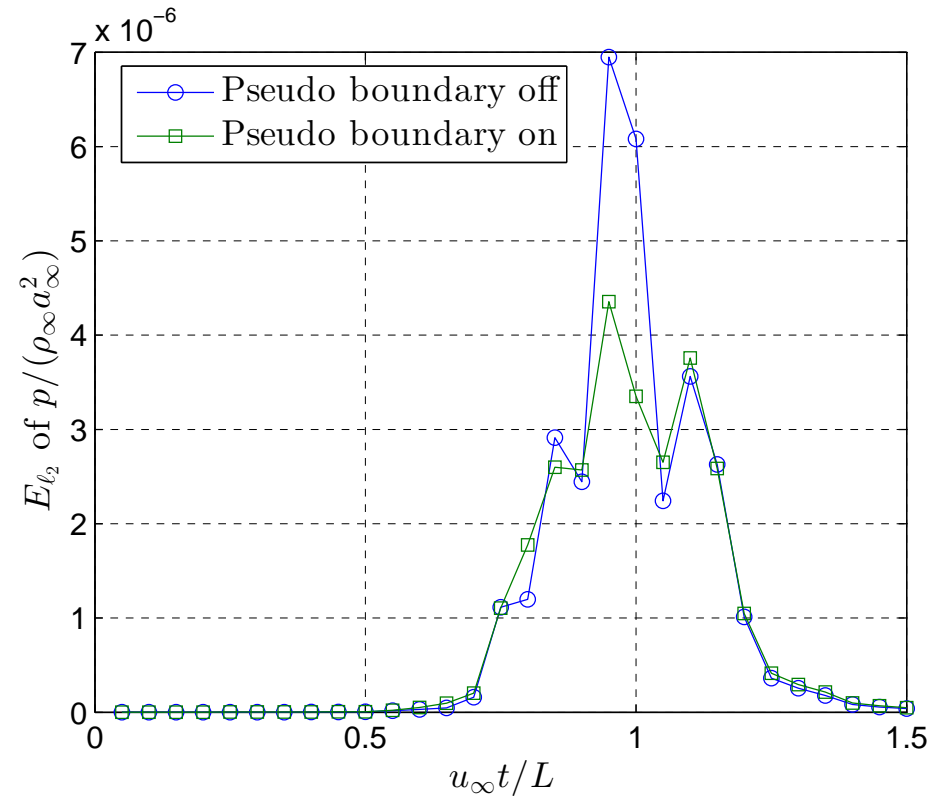

Figure 25: Time history of $\ell_{2}$-norm errors produced in the vortex convection problem based on normalised pressure $p /\left(\rho_{\infty} a_{\infty}^{2}\right)$. Result shown with and without pseudo-boundary schemes for $N \times N=60 \times 60$ and $\epsilon=5$ 
a variety of flow problems with varying degrees of linearity, on both uniform and curvilinear grids.

\section{Acknowledgement}

The authors would like to acknowledge the support of the Engineering and Physical Sciences Research Council under the Doctoral Training Centre grant $\mathrm{EP} / \mathrm{G} 03690 \mathrm{X} / 1$. All data supporting this study are openly available from the University of Southampton repository at http://dx.doi.org/10.5258/SOTON /399438. We would also like to thank the high performance computing facilities and supports services of the local IRIDIS-4 supercomputer at the University of Southampton, in the completion of this work.

\section{References}

[1] J. W. Kim, Optimised boundary compact finite difference schemes for computational aeroacoustics, Journal of Computational Physics 225 (2007) 995 - 1019.

[2] A. Sescu, R. Hixon, A. A. Afjeh, Multidimensional optimization of finite difference schemes for computational aeroacoustics, Journal of Computational Physics 227 (2008) 4563 - 4588.

[3] E. Johnsen, J. Larsson, A. V. Bhagatwala, W. H. Cabot, P. Moin, B. J. Olson, P. S. Rawat, S. K. Shankar, B. Sjgreen, H. Yee, X. Zhong, S. K. Lele, Assessment of high-resolution methods for numerical simulations of compressible turbulence with shock waves, Journal of Computational Physics 229 (2010) $1213-1237$.

[4] S. Kawai, K. S. Shankar, S. K. Lele, Assessment of localized artificial diffusivity scheme for large-eddy simulation of compressible turbulent flows, Journal of Computational Physics 229 (2010) 1739-1762.

[5] S. Nagarajan, S. K. Lele, J. H. Ferziger, A robust high-order compact method for large eddy simulation, Journal of Computational Physics 191 (2003) 392-419.

[6] E. Lamballais, V. Fortun, S. Laizet, Straightforward high-order numerical dissipation via the viscous term for direct and large eddy simulation, Journal of Computational Physics 230 (2011) $3270-3275$.

[7] Z.-S. Sun, Y.-X. Ren, C. Larricq, S.-Y. Zhang, Y.-C. Yang, A class of finite difference schemes with low dispersion and controllable dissipation for dns of compressible turbulence, Journal of Computational Physics 230 (2011) 4616 - 4635.

[8] A. W. Cook, J. J. Riley, Direct numerical simulation of a turbulent reactive plume on a parallel computer, Journal of Computational Physics 129 (1996) 263 - 283.

[9] J. W. Kim, D. J. Lee, Optimized compact finite difference schemes with maximum resolution, AIAA Journal 34 (1996) 887-893. 
[10] M. H. Carpenter, D. Gottlieb, S. Abarbanel, Stable and accurate boundary treatments for compact, high-order finite-difference schemes, Applied Numerical Mathematics 12 (1993) 55 - 87.

[11] Z. Liu, Q. Huang, Z. Zhao, J. Yuan, Optimized compact finite difference schemes with high accuracy and maximum resolution, International journal of aeroacoustics 7 (2008) 123-146.

[12] S. A. Jordan, The spatial resolution properties of composite compact finite differencing, Journal of Computational Physics 221 (2007) 558 - 576.

[13] S. A. Jordan, Optimization, resolution and application of composite compact finite difference templates, Applied Numerical Mathematics 61 (2011) 108 - 130.

[14] R. Vichnevetsky, J. B. Bowles, Fourier analysis of numerical approximations of hyperbolic equations, SIAM, 1982.

[15] R. L. Haupt, S. H. Haupt, Practical genetic algorithms, second ed., Wiley, 2004.

[16] J. W. Kim, High-order compact filters with variable cut-off wavenumber and stable boundary treatment, Computers \& Fluids 39 (2010) 1168 - 1182.

[17] S. K. Lele, Compact finite difference schemes with spectral-like resolution, Journal of Computational Physics 103 (1992) 16-42.

[18] C. K. W. Tam, Aliasing problem: category 1 problem 1 analytic solutions, in: Fourth computational aeroacoustics workshop on benchmark problems, NASA Glenn Research Centre, Cleveland. USA, 2003, pp. 31-32.

[19] H. C. Yee, N. D. Sandham, M. J. Djomehri, Low-dissipative high-order shock-capturing methods using characteristic-based filters, Journal of Computational Physics 150 (1999) 199 - 238.

[20] J. W. Kim, D. J. Lee, Generalized characteristic boundary conditions for computational aeroacoustics, AIAA Journal 38 (2000) 2040-2049.

[21] D. V. Gaitonde, M. R. Visbal, Pade-type higher-order boundary filters for the navier-stokes equations, Aiaa Journal 38 (2000) 2103-2112.

\section{Appendix A.}

An exact solution to the second benchmark problem is usually obtained by substituting $\hat{x}=x-u_{\infty} t$ for $x$ in Eq. (34). However, for more non-linear cases it appears this formulation may not be the most appropriate when analysing the performance of the boundary schemes. This was made apparent by the presence of an additional error which manifested itself well before the vortex reaches the domain boundary, masking the true performance of the schemes. This is shown graphically by Figure A.26, which contains ab- 
solute error contours based on the exact solution for normalised pressure $\left(|e|=\left|\left(p-p_{\text {exact }}\right) /\left(\rho_{\infty} a_{\infty}^{2}\right)\right|\right)$ plotted with three contour level bandwidths, and $\epsilon=5$. As the contour bandwidth is decreased it becomes clear that the most substantial error actually exists near the vortex core, and persists until the vortex has left the domain. If errors from the exact solution are alternatively quantified in terms of an $\ell_{2}$-norm, the influence of this effect can not be detected until an error level of approximately $10^{-6}$ is obtained, requiring a grid density close to $N \times N=600 \times 600$. Therefore it is expected that this issue has not been encountered in prior optimisation attempts, due to relatively higher peak errors obtained during the simulation. Since this issue is only encountered for non-linear vortex strengths (above $\epsilon=0.1$ ) it seems likely that it is caused by some non-linear physical phenomenon which is successfully detected in the simulation, but not properly represented by the analytical solution due to assumptions made in its derivation. For this reason numerical errors generated by the boundary schemes were analysed by comparing solutions obtained on both a full-size and truncated domain. The full-size domain resolving the vortex solely with interior points, while the truncated domain requires the vortex to pass through an exit boundary where the boundary schemes are implemented. 


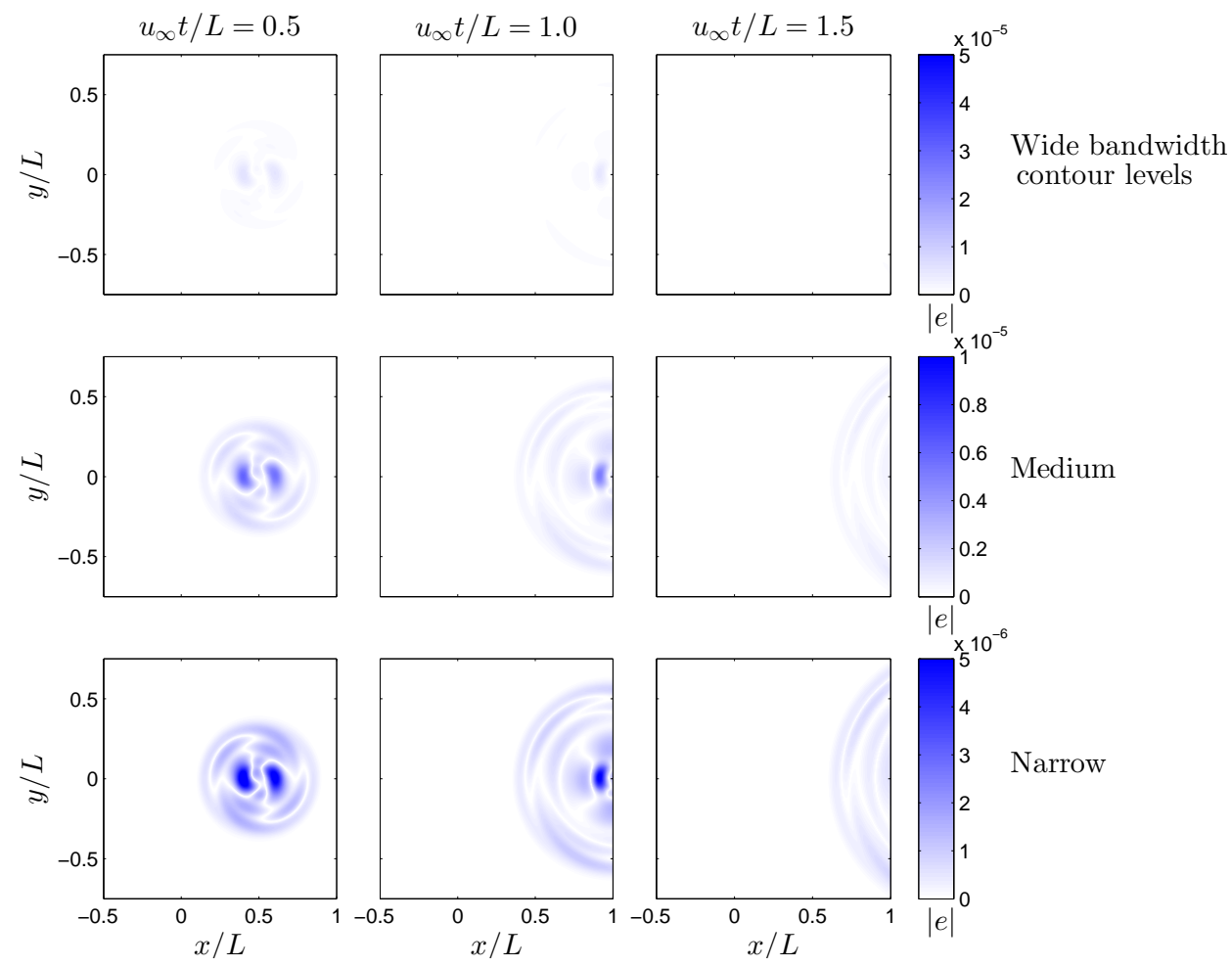

Figure A.26: Absolute error contours based on the exact solution for normalised pressure, $|e|=\left|\left(p-p_{\text {exact }}\right) /\left(\rho_{\infty} a_{\infty}^{2}\right)\right|$. Calculated with $\mathrm{CFL}=0.5$ and $\epsilon=5$ with $N \times N=300 \times 300$. Errors are shown at three instances of time and with three contour level bandwidths. Top: wide bandwidth $5 \times 10^{-5}$, Middle: medium bandwidth $1 \times 10^{-5}$, Bottom: narrow bandwidth $5 \times 10^{-6}$ 